\title{
On a Navier-Stokes-Allen-Cahn model with inertial effects
}

\author{
Gianluca Favre \\ Faculty of Mathematics, University of Vienna \\ Oskar-Morgenstern-Platz 1, 1090 Wien, Austria \\ E-mail: gianluca.favre@univie.ac.at \\ Giulio Schimperna \\ Dipartimento di Matematica, Università di Pavia, \\ Via Ferrata 5, I-27100 Pavia, Italy \\ E-mail: giusch04@unipv.it
}

March 19, 2019

\begin{abstract}
A mathematical model describing the flow of two-phase fluids in a bounded container $\Omega$ is considered under the assumption that the phase transition is influenced by inertial effects. The model couples a variant of the Navier-Stokes system for the velocity $\mathbf{u}$ with an Allen-Cahn-type equation for the order parameter $\varphi$ relaxed in time in order to introduce inertia at the microscopic level. The resulting model is characterized by second-order material derivatives which constitute the main difficulty in the mathematical analysis. In order to obtain a tractable problem, a viscous relaxation term is then included in the phase equation. The mathematical results consist in existence of weak solutions in 3D and, under additional assumptions, existence and uniqueness of strong solutions in 2D. A partial characterization of the long-time behavior of solutions is also given and in particular some issues related to dissipation of energy are discussed.
\end{abstract}

Keywords: two-phase fluid, Allen-Cahn, Navier-Stokes, inertial effects, viscous relaxation, existence and uniqueness.

AMS (MOS) subject classification: 35Q35, 35K10, 35L82, 76D05, 80A22.

\section{Introduction}

In this paper we are concerned with the mathematical analysis of the following PDE system describing the evolution of a phase-changing fluid through the variables $\mathbf{u}$ (macroscopic velocity of the flow) and $\varphi$ (order parameter, normalized in such a way that the values $\varphi= \pm 1$ represent the pure states):

$$
\begin{aligned}
& \mathbf{u}_{t}+\mathbf{u} \cdot \nabla \mathbf{u}+\nabla p-\Delta \mathbf{u}=-\operatorname{div}(\nabla \varphi \otimes \nabla \varphi) \\
& \operatorname{div} \mathbf{u}=0 \\
& \pi=\varphi_{t}+\mathbf{u} \cdot \nabla \varphi-\epsilon \Delta \varphi+\sigma \varphi \\
& \kappa \pi_{t}+\delta \mathbf{u} \cdot \nabla \pi+\pi-\Delta \varphi+f(\varphi)=0
\end{aligned}
$$

with parameters $\kappa>0, \delta \geq 0, \sigma \geq 0$ and $\epsilon>0$ whose role will be clarified in the sequel. The evolution is assumed to take place in a bounded domain $\Omega \subset \mathbb{R}^{d}, d \in\{2,3\}$, over a bounded but otherwise arbitrary time reference interval $(0, T)$. More precisely, in order to avoid complications related with the interaction with the boundary, we will assume that $\Omega$ is the unitary flat torus $\Omega \sim$ $[0,1]^{d}$. Correspondingly, we will take periodic boundary conditions for all variables. This may be seen as the simplest situation where one can attempt a mathematical analysis of the system; we however remark that at least a part of our results could be extended to other types of boundary conditions at the price of some additional technicality in the proofs. 
Relation (1.1), with the incompressibility constraint (1.2), is the standard Navier-Stokes system where the right-hand side of (1.1) accounts for the extra-stress due to the effects of phase transition. On the other hand, the change of phase is governed by a proper version of the Allen-Cahn equation, where inertial effects are taken into account. Indeed, from a mechanical point of view, the phase transition phenomenon may be thought to be driven by the microscopic motion of molecules (we refer to the monograph [21] for a general discussion), which motivates the occurrence of an inertial term leading to a finite propagation speed of the phase transition front. The function $f$ in (1.4) represents the derivative of a configuration potential $F$ of double-well type whose minima are attained in correspondence of the pure phases of the material. Mathematically speaking, $F$ will be assumed to have a polynomial growth at infinity (of arbitrary order) and to be $\lambda$-convex (cf. (2.5) below). It is worth remarking that, even in dimension $d=2$, the polynomial behavior at infinity seems to be necessary in order for our arguments to work; in particular, our results do not extend to more general (e.g., logarithmic) potentials.

In the above formulation, the Allen-Cahn equation for the phase parameter has been split into the coupled relations (1.3), (1.4). Indeed, this choice seems more natural because it gives a better description of the transport effects in terms of the auxiliary variable $\pi$, which plays an important role from both the physical and the mathematical viewpoint. Indeed, from (1.3) we can see that $\pi$ represents a sort of regularization of the material derivative of $\varphi, \epsilon$ and $\sigma$ being the (small) regularization parameters. The reasons for considering such regularization terms are shortly discussed in the next paragraphs, whereas a much more detailed explanation (also considering the physical implications) is given in Section 6] below. Here, it is also worth observing that, for $\kappa=\delta>0$, equation (1.4) includes the term $\pi_{t}+\mathbf{u} \cdot \nabla \pi$, which, by (1.3), can be interpreted as a regularization of the second material derivative of $\varphi$.

The mathematical literature devoted to the study of models for complex (i.e., two-phase or two-component) fluids is very vast. Here we limit ourselves to describe a limited number of contributions without any claim of completeness. The bibliographic entries in the papers mentioned below can provide the reader with a further set of references. The first article presenting a mathematical model for two-phase fluids, dating back to 1977, is [28, where the so-called "H-model" for binary fluids was introduced. Actually, the approach devised there is still at the base of more modern models. Later, in 1996, Gurtin et al. [27] introduced a coupled Navier-Stokes-Cahn-Hilliard whose physical derivation was obtained using a balance law for microforces in conjunction with constitutive equations consistent with a mechanical version of the second law of Thermodynamics. This mechanical interpretation of the phase transition effect as an action of microscopic forces is also at the base of the present model and, in particular, justifies inertial effects at the microscopic level. A comprehensive presentation of mechanically-based phase change models is given in the monograph 21. A situation where the (compressible) Navier-Stokes system is coupled with an equation of Allen-Cahn type was then introduced in [6] in order to provide a mathematical description of the physical problem of cavitation in a flowing fluid. In particular, in that contribution, a Korteweg term having the same expression $-\operatorname{div}(\nabla \varphi \otimes \nabla \varphi)$ as in our (1.1) appears as a forcing term on the right-hand side of the Navier-Stokes system.

The above mentioned papers are mainly devoted to presenting a number of significant models and discussing their compatibility with the basic laws of Thermodynamics and Fluid Mechanics. Speaking, instead, of mathematical results, the literature devoted to the analysis (also from the numerical viewpoint) of models for complex fluids is more recent. In 2003, Liu and Shen 31 provided a variational formulation of a Cahn-Hilliard-Navier-Stokes for binary fluids with matched densities and viscosities and proved analytical and numerical results partly based on a Fourier-spectral method. In 2009, Abels [1] studied a similar model coupling the incompressible Navier-Stokes system with the Cahn-Hilliard equation and proved existence both of weak and of strong solutions under natural conditions on data. In particular he considered the case when the two fluids have the same density but may have different viscosities. He also discussed the sharp-interface limit of the resulting model. A further related contribution [2] is devoted to the analysis of a Navier-Stokes-Mullins-Sekerka model. Another important class of results is devoted to studying the long-time properties of solutions; among the many related works, we may mention [36, where the long time behavior of the strong solution to a model of viscous incompressible fluids is analyzed. In particular the rate of the convergence to equilibria is established there. More recently, in [23, the existence of global and exponential attractors has been established for a Cahn-Hilliard-Navier-Stokes model in two space dimensions. Finally, we 
may mention [11, where the global regularity of strong solutions to a Cahn-Hilliard-Navier-Stokes model with mixed partial viscosity and mobility is analyzed.

Second order in time (hyperbolic or pseudo-parabolic) relaxations of the Allen-Cahn and CahnHilliard equations have been considered in several different contexts and under various mathematical assumptions. We may mention, without any claim of completeness, the papers [8, 24, 25, 26] (see also the references therein). In most of these contributions, thermal effects have also been considered, leading to the so-called phase-field models with inertia. Inertial effects at the microscopic level have been observed also in (nematic) liquid crystals (see, e.g., [18, 22]). On the other hand, at least up to our knowledge, phase transition models with inertia have not been studied so far in the case of two-phase fluids, i.e., when the process is influenced by a macroscopic velocity satisfying some version of the Navier-Stokes system.

Coming to a description of our results, we first observe that the term $-\epsilon \Delta \varphi$ in (1.3), acts as a viscous regularization of the phase variable. This type of effect may be experimentally observed at least as far as $\epsilon>0$ is very small, and may correspond to a strong damping in the phase transition. On the other hand, here such term is added mainly for mathematical reasons (i.e., it acts as a smoothing parameter). Indeed, omitting it (i.e., taking $\epsilon=0$ ), the resulting system seems to present, even in $2 \mathrm{D}$, insurmountable mathematical difficulties, especially related to the control of the right-hand side of (1.1) (see also Remark 5 below for additional comments).

Our existence proof will be based on a standard Faedo-Galerkin regularization scheme, combined with the derivation of a priori estimates and compactness arguments to pass to the limit. Entering details, we start observing that the only a priori information that is always guaranteed for solutions to our system is the energy estimate, which is a direct consequence of the variational structure of the model (cf. Subsec. 4.1 below for more details). On the other hand, this bound is sufficient only for proving existence of weak solutions. In particular, it is only thanks to the viscous regularization in (1.3) that we can obtain some information on the second space derivatives of $\varphi$. Actually, for $\epsilon=0$ taking the limit of the right-hand side of (1.1) would likely be hopeless due to lack of compactness. Indeed, here it does not seem possible to obtain the strong $L^{2}$-convergence of $\nabla \varphi$ via contractive arguments (as is usually done for the decoupled semilinear wave equation with subcritical nonlinearity), due to the presence of the transport terms.

Once we have obtained existence of weak solutions (which we can do both in 2D and in 3D) looking for additional properties is more difficult, even in the $2 \mathrm{D}$ case, and we can only prove a number of partial results holding under additional regularity assumptions on coefficients and data. Entering details, in 2D we can try to construct strong solutions (such a classification is mutuated by the standard terminology used for Navier-Stokes) and, indeed, we can prove their existence, but only in the case when the second order transport term is neglected, i.e., one has $\delta=0$ in (1.4). In the class of strong solutions one can also prove uniqueness. This result holds, with a conditional nature, also in 3D (where existence of strong solutions is not known, of course), or for $\delta>0$.

A further question we address is related to energy dissipation. Actually, due to the absence of external sources and to the choice of periodic boundary conditions, one expects that at least a part of the kinetic and chemical energy of the body is gradually converted into heat. On the other hand, we can explicitly prove this fact only in two cases, i.e. when either $\sigma>0$ in (1.3) or $\delta=\kappa$ in (1.4). Actually, if $\sigma>0$ one can control, uniformly in time, the (possibly nonpositive) contribution coming from the combination of the additional viscosity term $-\epsilon \Delta \varphi$ with the non-monotone semilinear term $f(\varphi)$. On the other hand, for $\delta=\kappa$, corresponding to the situation when the true material derivative of $\pi$ occurs in (1.4), dissipativity holds even for $\sigma=0$ thanks to a cancellation property. Under different choices on regularization coefficient, dissipativity of energy may actually not hold. This (somehow unexpected) behavior is correlated with the presence of the viscous regularization parameter $\epsilon$ in (1.3). A discussion of this issue, and of its physical implications, is given in Section 6 below. There, we also mention the possibility of considering, in place of $\sigma \varphi$, a more general semilinear contribution $\sigma(\varphi)$ in (1.3) (with one relevant choice being given by $\sigma(\cdot)=f(\cdot))$. This term, at least under suitable conditions on the function $\sigma$, would describe some form of nonlinear damping effect (see, e.g., 5, 7, 12, 14, 15, 29, 30, 34, for related models); the resulting mathematical problem will be possibly addressed in a forthcoming paper.

It is finally worth noting that, in the language of dynamical systems, uniform dissipativity of energy implies the existence of a bounded absorbing set in a suitable phase-space, a fact that may serve 
as a starting point for studying the long-time behavior of solution trajectories and proving existence of attractors. These issues will be also possibly addressed in a future work.

We conclude with the plan of the paper. The next section is devoted to presenting our precise mathematical assumptions on coefficients and data and a number of preliminary considerations. Then, our main results are stated in the subsequent Section 3 . The core of the proofs, including the basic a priori estimates and the arguments used for passing to the limit in the approximation, are then presented in Section 4. A possible construction of regularized solutions by means of a FaedoGalerkin scheme is given in Section 5. Finally, in Section 6, we provide some final comments and, in particular, we discuss a bit more extensively the physical implications of our choices about regularizing parameters.

\section{Preliminaries}

\subsection{Notation and functional setup}

We will note as $\Omega$ the unit flat torus $[0,1]^{d}$, with $d=2$ or $d=3$. As is customary, all functions defined on $\Omega$ will be implicitly assumed to satisfy periodic boundary conditions in a suitable sense. This will not be emphasized in the notation, for the sake of simplicity. For instance, we will set $H:=L^{2}(\Omega)$ and $V:=H^{1}(\Omega)$ implicitly assuming $\Omega$-periodicity. These spaces will be used as function spaces for the phase variable; the same symbols $H$ and $V$ will be used also for denoting vector- or tensor-valued functions (we may write, for instance, $\nabla \varphi \in H$ ). The standard scalar product in $H$ will be noted as $(\cdot, \cdot)$. Since the immersion $V \subset H$ is continuous and dense, identifying $H$ with $H^{\prime}$ through the above scalar product we obtain the Hilbert triplet $\left(V, H, V^{\prime}\right)$ for the phase variable.

Concerning the velocity function $\mathbf{u}$, we set

$$
\mathbf{C}_{\mathrm{div}}^{\infty}(\Omega)=\left\{\mathbf{u} \in\left[C^{\infty}(\Omega)\right]^{d}: \operatorname{div} \mathbf{u}=0\right\}, \quad V_{\mathrm{div}}:={\overline{\mathbf{C}_{\mathrm{div}}^{\infty}(\Omega)}}^{H^{1}(\Omega)}, \quad H_{\mathrm{div}}:={\overline{\mathbf{C}_{\mathrm{div}}^{\infty}(\Omega)}}^{L^{2}(\Omega)},
$$

where, again, $\Omega$-periodicity is still implicitly subsumed everywhere. The spaces $H_{\mathrm{div}}$ and $V_{\mathrm{div}}$ are seen as (closed) subspaces of $H$ and $V$ (more precisely, of $H^{d}$ and $V^{d}$ ), respectively, and in particular they are endowed with the corresponding norms. Then, the embedding $V_{\text {div }} \subset H_{\text {div }}$ is continuous and dense, which permits us to identify $V_{\text {div }}$ with its topological dual $V_{\text {div }}^{\prime}$ by means of the scalar product of $H_{\text {div }}$, still denoted by $(\cdot, \cdot)$, and to construct the "velocity Hilbert triplet" $\left(V_{\text {div }}, H_{\text {div }}, V_{\text {div }}^{\prime}\right)$. We recall that $V_{\text {div }}$ is endowed with the bilinear form $((\mathbf{u}, \mathbf{v})):=(\nabla \mathbf{u}, \nabla \mathbf{v})$ for all $\mathbf{u}, \mathbf{v} \in V_{\mathrm{div}}$. Moreover, given a generic Banach space $X$ (and particularly in the cases $X=V, X=V_{\text {div }}$ ), we will denote by $\langle\cdot, \cdot\rangle$ the duality between $X^{\prime}$ and $X$. As is customary, we define the trilinear $V_{\text {div }}$-continuous form

$$
b(\mathbf{u}, \mathbf{v}, \mathbf{w})=\int_{\Omega}(\mathbf{u} \cdot \nabla \mathbf{v}) \cdot \mathbf{w} \mathrm{d} x \quad \forall \mathbf{u}, \mathbf{v}, \mathbf{w} \in V_{\mathrm{div}}
$$

and the bilinear form $\mathbf{B}: V_{\mathrm{div}} \times V_{\mathrm{div}} \rightarrow V_{\mathrm{div}}^{\prime}$ given by

$$
\langle\mathbf{B}(\mathbf{u}, \mathbf{v}), \mathbf{w}\rangle=b(\mathbf{u}, \mathbf{v}, \mathbf{w}) \quad \forall \mathbf{u}, \mathbf{v}, \mathbf{w} \in V_{\text {div }} .
$$

Using Ladyzhenskaya's inequality (see, e.g. [33, Chap. 5]), it turns out that the following properties hold for every $\mathbf{u}, \mathbf{v}$ and $\mathbf{w} \in V_{\text {div }}$ :

$$
\begin{gathered}
|b(\mathbf{u}, \mathbf{v}, \mathbf{w})| \leq c\|\mathbf{u}\|_{H_{\mathrm{div}}}^{1 / 2}\|\mathbf{u}\|_{V_{\mathrm{div}}}^{1 / 2}\|\mathbf{v}\|_{V_{\mathrm{div}}}\|\mathbf{w}\|_{V_{\mathrm{div}}} \quad \text { if } d=3, \\
|b(\mathbf{u}, \mathbf{v}, \mathbf{w})| \leq c\|\mathbf{u}\|_{H_{\mathrm{div}}}^{1 / 2}\|\mathbf{u}\|_{V_{\mathrm{div}}}^{1 / 2}\|\mathbf{v}\|_{V_{\mathrm{div}}}\|\mathbf{w}\|_{H_{\mathrm{div}}}^{1 / 2}\|\mathbf{w}\|_{V_{\mathrm{div}}}^{1 / 2} \quad \text { if } d=2 .
\end{gathered}
$$

\subsection{Assumptions on the potential}

We let $F \in C^{2}(\mathbb{R} ; \mathbb{R})$ and setting $f:=F^{\prime}$ we assume that

$$
K_{0}\left(|s|^{p}+1\right) \geq f^{\prime}(s) \geq k_{0}|s|^{p}-\lambda_{0}
$$

for some $K_{0}, k_{0}, p>0, \lambda_{0} \geq 0$ and every $s \in \mathbb{R}$. Namely, $f$ grows at infinity as a power-like function of exponent $p+1>1$ (i.e., it is strictly superlinear). Note that the above implies in particular that 
$F$ is (at least) $\lambda$-convex, i.e., $F^{\prime \prime}=f^{\prime}$ is everywhere greater than $-\lambda_{0}$, with true convexity holding in the case $\lambda_{0}=0$.

A practical and common example of a phase potential satisfying the above is the standard double well potential having the expression $F(s)=\left(s^{2}-1\right)^{2}$ for which (2.5) holds of course for $p=2$.

The above relation has several useful consequences, some of which are listed below. First, by integration one can easily deduce

$$
K^{\prime}\left(|s|^{p+1}+1\right) \geq f(s) \operatorname{sign} s \geq k^{\prime}|s|^{p+1}-\lambda_{1},
$$

and, integrating again,

$$
K^{\prime \prime}\left(|s|^{p+2}+1\right) \geq F(s) \geq k^{\prime \prime}|s|^{p+2}-\lambda_{2},
$$

where $K^{\prime}, k^{\prime}, K^{\prime \prime}, k^{\prime \prime}>0$ and $\lambda_{1}, \lambda_{2} \geq 0$ are computable constants depending only on $K_{0}, k_{0}, p, \lambda_{0}$. Moreover, combining (2.6) and (2.7), it is not difficult to deduce that

$$
k_{p}\left(|s|^{p+2}+F(s)\right)-c_{p} \leq f(s) s \leq C_{p}\left(|s|^{p+2}+1\right) \quad \forall s \in \mathbb{R},
$$

where the constants $k_{p}, C_{p}>0$ and $c_{p} \geq 0$ depend only on the given values of the parameters in (2.5). Moreover, thanks to $p+2>2$, we also observe that for any (large) $M>0$ and (small) $\varepsilon>0$ there exists $c(M, \varepsilon)>0$ such that

$$
M|s|^{2} \leq \varepsilon F(s)+c(M, \varepsilon) \quad \forall s \in \mathbb{R}
$$

\subsection{Initial data and conservation properties}

In the sequel we will note the spatial mean of a generic function $v$ defined over $\Omega$ as

$$
v_{\Omega}:=\frac{1}{|\Omega|} \int_{\Omega} v \mathrm{~d} x=\int_{\Omega} v \mathrm{~d} x
$$

the second equality holding because $\Omega$ is the unit torus. We also recall the Poincaré-Wirtinger inequality

$$
\left\|v-v_{\Omega}\right\|_{H} \leq c\|\nabla v\|_{H}
$$

holding for any $v \in V$ and for some $c>0$. In particular, thanks to periodic boundary conditions, the above holds with $v_{\Omega}=0$ when $v=D_{x_{i}} z$ for some $z \in H^{2}(\Omega)$ and $i \in\{1, \ldots, d\}$.

We observe that, due to the choice of periodic boundary conditions, any hypothetical solution to our problem satisfies some conservation properties. First of all, integrating (1.1) over $\Omega$, we actually have

$$
\frac{\mathrm{d}}{\mathrm{d} t} \mathbf{u}_{\Omega}=0
$$

i.e., the mean value of the velocity is conserved in time. Such a property corresponds to the conservation of (total) momentum in absence of external forces.

Then, in order to reduce technical complications we shall always assume that

$$
\mathbf{u}_{0} \in H_{\text {div }}, \quad\left(\mathbf{u}_{0}\right)_{\Omega}=0 .
$$

Indeed the case when $\left(\mathbf{u}_{0}\right)_{\Omega} \neq 0$ could be reduced to the present one by rewriting the system in terms of the translated variable $\mathbf{u}-\mathbf{u}_{\Omega}$.

Next, integrating (1.3) and (1.4), we deduce, respectively,

$$
\begin{aligned}
& \frac{\mathrm{d}}{\mathrm{d} t} \varphi_{\Omega}+\sigma \varphi_{\Omega}=\pi_{\Omega}, \\
& \kappa \frac{\mathrm{d}}{\mathrm{d} t} \pi_{\Omega}+\pi_{\Omega}+\int_{\Omega} f(\varphi) \mathrm{d} x=0,
\end{aligned}
$$

whence, combining the above relations, we obtain (here primes denote derivation in time)

$$
\kappa\left(\varphi_{\Omega}\right)^{\prime \prime}+(1+\kappa \sigma)\left(\varphi_{\Omega}\right)^{\prime}+\sigma \varphi_{\Omega}+\int_{\Omega} f(\varphi) \mathrm{d} x=0
$$

This relation rules the evolution of the total mass of either component of the binary fluid. Note, however, that a decay property for $\varphi_{\Omega}$ does not follow directly from (2.16) due to the presence of the nonlinear function $f$. 


\section{Main Results}

Basically we can distinguish our results into two classes. The first one refers to the regularity setting of weak solutions (this essentially means that the initial data have the sole regularity corresponding to the finiteness of the physical energy). In such a framework, we can prove existence both in 2D and in 3D (Theorem 1) for $\delta \geq 0$. Moreover, in all cases except when $\sigma=0$ and $\delta \neq \kappa$, we can also prove an energy dissipation principle (Prop. 21), i.e. the fact that, whatever is the magnitude of the energy at the initial time, solution trajectories (or, at least those solution trajectories that arise as cluster points of approximating families; indeed, uniqueness is not known at this level) tend to have an energy configuration that is below some computable threshold depending only on the parameters of the system (and not on the magnitude of the initial data). In the language of dynamical systems this corresponds to the existence of a uniformly absorbing set. The reasons why we are able to prove such a condition only under additional assumptions on coefficients are detailed in Section 6 below.

The second family of results refer to the class of strong solutions, hence requiring some more smoothness of initial data. Unfortunately, even in 2D, we can prove existence of such solutions (Theorem 3) only in the case when $\delta=0$, i.e. the second-order transport effect is neglected. In this case, we can also prove uniqueness of strong solutions (Theorem 4). The result holds both in 2D and in $3 \mathrm{D}$, having of course a conditional nature in the latter case.

That said, we start with stating our first result devoted to existence of weak solutions:

Theorem 1 (Existence of weak solutions, $d=2,3$ ). Let $d \in\{2,3\}$, let $\epsilon>0, \sigma \geq 0, \kappa>0$ and $\delta \geq 0$. Let also Assumption (2.5) hold. Let $T>0$ and let the initial data satisfy

$$
\boldsymbol{u}_{0} \in H_{\mathrm{div}}, \quad\left(\boldsymbol{u}_{0}\right)_{\Omega}=0, \quad \varphi_{0} \in V \cap L^{p+2}(\Omega), \quad \pi_{0} \in H .
$$

Then there exists at least one triple $(\boldsymbol{u}, \varphi, \pi)$ belonging to the regularity class

$$
\begin{aligned}
& \boldsymbol{u} \in W^{1, q}\left(0, T ; V_{\mathrm{div}}^{\prime}\right) \cap L^{\infty}\left(0, T ; H_{\mathrm{div}}\right) \cap L^{2}\left(0, T ; V_{\mathrm{div}}\right), \\
& \varphi \in L^{\infty}(0, T ; V) \cap L^{\infty}\left(0, T ; L^{p+2}(\Omega)\right) \cap L^{2}\left(0, T ; H^{2}(\Omega)\right), \\
& \varphi_{t} \in L^{2}\left(0, T ; L^{s}(\Omega)\right), \\
& \pi \in L^{\infty}(0, T ; H), \\
& \pi_{t} \in L^{2}\left(0, T ;\left(W^{1,3}\right)^{\prime}(\Omega)\right),
\end{aligned}
$$

where

$$
q=s=2 \text { if } d=2, \quad q=4 / 3, s=3 / 2 \text { if } d=3,
$$

satisfying system (1.1) -(1.4) in the following weak sense

$$
\begin{aligned}
& \left\langle\boldsymbol{u}_{t}, \boldsymbol{v}\right\rangle-\int_{\Omega}(\boldsymbol{u} \otimes \boldsymbol{u}): \nabla \boldsymbol{v} \mathrm{d} x+(\nabla \boldsymbol{u}, \nabla \boldsymbol{v})=\int_{\Omega}(\nabla \varphi \otimes \nabla \varphi): \nabla \boldsymbol{v} \mathrm{d} x, \\
& \pi=\varphi_{t}+\boldsymbol{u} \cdot \nabla \varphi-\epsilon \Delta \varphi+\sigma \varphi, \quad \text { a.e. } i n \Omega \\
& \kappa\left\langle\pi_{t}, v\right\rangle+(\pi, v)-\delta \int_{\Omega} \pi \boldsymbol{u} \cdot \nabla v \mathrm{~d} x+(\nabla \varphi, \nabla v)+\int_{\Omega} f(\varphi) v \mathrm{~d} x=0,
\end{aligned}
$$

for almost every $t \in(0, T)$, every $\boldsymbol{v} \in V_{\mathrm{div}}$ and every $v \in W^{1,3}(\Omega)$, and complying with the initial conditions

$$
\left.\boldsymbol{u}\right|_{t=0}=\boldsymbol{u}_{0},\left.\quad \varphi\right|_{t=0}=\varphi_{0},\left.\quad \pi\right|_{t=0}=\pi_{0}
$$

almost everywhere in $\Omega$. The triple $(\boldsymbol{u}, \varphi, \pi)$ will be noted as a weak solution in the sequel.

Remark 1. It is worth observing that condition (3.1) corresponds exactly to the finiteness of the physical energy (cf. (4.7) below) at the initial time. Indeed, assumption $\varphi_{0} \in L^{p+2}(\Omega)$ is equivalent to asking $F\left(\varphi_{0}\right) \in L^{1}(\Omega)$ due to (2.5).

Since $T>0$ is arbitrary, we can assume that the weak solutions provided by Theorem 1 could be extended to be defined for any time $t \in[0, \infty)$. In this perspective we can prove the following 
Proposition 2 (Dissipativity). Let the hypotheses of Theorem 1 hold and let us additionally assume that, either $\sigma>0$, or $\delta=\kappa$. Then there exists a constant $C_{0}$ independent of the initial data (but depending on the other parameters of the system) and a time $T_{0}$ depending only on the "energy" of the initial datum, i.e. on the quantity

$$
\left\|\boldsymbol{u}_{0}\right\|_{H_{\mathrm{div}}}+\left\|\varphi_{0}\right\|_{V}+\left\|\varphi_{0}\right\|_{L^{p+2}(\Omega)}+\left\|\pi_{0}\right\|_{H},
$$

such that any weak solution provided by Theorem 1 emanating from this initial datum satisfies

$$
\|\boldsymbol{u}(t)\|_{H_{\mathrm{div}}}+\|\varphi(t)\|_{V}+\|\varphi(t)\|_{L^{p+2}(\Omega)}+\|\pi(t)\|_{H} \leq C_{0} \quad \forall t \geq T_{0}
$$

Remark 2. In the terminology of dynamical systems, the constant $C_{0}$ in estimate (3.13) may be interpreted as the "radius" of an absorbing set with respect to the norms specified there (which in turn somehow quantify the magnitude of the energy).

Remark 3. Since the uniqueness of weak solutions is not known at this level, the dissipativity property in (3.13) has to be carefully interpreted. Indeed, its proof is obtained by passing to the limit in an analogue relation holding at the approximate level (i.e., for some regularized solution that has better properties). Hence, any weak solution that is a limit point of a sequence of approximate solutions turns out to satisfy it. On the other hand, we cannot exclude that there might exist "bad" weak solutions, unrelated to the approximation scheme, that do not satisfy (3.13).

Theorem 3 (Existence of strong solutions). Let $d=2, \epsilon>0, \kappa>0, \delta=0, \sigma \geq 0$ and let Assumption (2.5) hold. Let also $T>0$ and let the initial data satisfy

$$
\boldsymbol{u}_{0} \in V_{\mathrm{div}}, \quad\left(\boldsymbol{u}_{0}\right)_{\Omega}=0, \quad \varphi_{0} \in H^{2}(\Omega), \quad \pi_{0} \in V
$$

Then there exists almost one strong solution, namely a triple $(\boldsymbol{u}, \varphi, \pi)$ with

$$
\begin{aligned}
& \boldsymbol{u} \in H^{1}\left(0, T ; H_{\mathrm{div}}\right) \cap L^{\infty}\left(0, T ; V_{\mathrm{div}}\right) \cap L^{2}\left(0, T ; H_{\mathrm{div}}^{2}(\Omega)\right), \\
& \varphi \in H^{1}(0, T ; V) \cap L^{\infty}\left(0, T ; H^{2}(\Omega)\right) \cap L^{2}\left(0, T ; H^{3}(\Omega)\right), \\
& \pi \in H^{1}(0, T ; H) \cap L^{\infty}(0, T ; V),
\end{aligned}
$$

satisfying system (1.1)-(1.4) in the following sense:

$$
\begin{aligned}
& \boldsymbol{u}_{t}+\operatorname{div}(\boldsymbol{u} \otimes \boldsymbol{u})+\nabla p+\operatorname{div}(\nabla \varphi \otimes \nabla \varphi)-\Delta \boldsymbol{u}=0 \quad \text { a.e. in }(0, T) \times \Omega, \\
& \operatorname{div} \boldsymbol{u}=0 \quad \text { a.e. in }(0, T) \times \Omega, \\
& \pi=\varphi_{t}+\boldsymbol{u} \cdot \nabla \varphi-\epsilon \Delta \varphi+\sigma \varphi \quad \text { a.e. in }(0, T) \times \Omega, \\
& \kappa \pi_{t}+\pi-\Delta \varphi+f(\varphi)=0 \quad \text { a.e. in }(0, T) \times \Omega
\end{aligned}
$$

and the initial conditions as in (3.11).

Theorem 4 (Uniqueness of strong solutions). Let $d \in\{2,3\}, \sigma \geq 0, \kappa>0, \epsilon>0$ and let Assumption (2.5) hold. Let also $T>0$ and let $\left(\boldsymbol{u}_{i}, \varphi_{i}, \pi_{i}\right), i=1,2$ be a couple of strong solutions (in the sense of the previous theorem, and satisfying in particular the regularity conditions (3.15)-(3.17)) emanating from the same initial datum $\left(\boldsymbol{u}_{0}, \varphi_{0}, \pi_{0}\right)$ satisfying (3.14). Moreover, let either $\delta=0$ or $\delta>0$ together with the additional assumption

$$
\pi_{i} \in L^{2}\left(0, T ; W^{1,3}(\Omega)\right) \quad \text { for some } i \in\{1,2\} .
$$

Then $\left(\boldsymbol{u}_{1}, \varphi_{1}, \pi_{1}\right)=\left(\boldsymbol{u}_{2}, \varphi_{2}, \pi_{2}\right)$ almost everywhere in $(0, T) \times \Omega$.

Remark 4. The uniqueness result is conditional in two aspects. First of all, existence of strong solutions clearly cannot be obtained for $d=3$. Moreover, for $\delta>0$ (a case which is also not covered by Theorem 3), we can prove that two hypothetical strong solutions emanating from the same initial datum coincide only in the case when either of the two satisfies the additional regularity (3.22). 


\section{Proofs}

In this section we will prove Theorems 1, 3, 4 as well as Proposition 2, We start presenting a number of basic a priori estimates by working directly, though formally, on system (1.1)-(1.4) without referring explicitly to any regularization or approximation. Actually, the variational structure underlying system (1.1)-(1.4) is rather simple and for this reason it seems to be worth starting with the computations leading to the energy inequality. This actually constitutes the main a priori information that any (reasonably defined) solution should satisfy. Then, a possible approach via a Faedo-Galerkin regularization compatible with the a priori estimates will be outlined in Section 5 below.

In what follows we will note as $c$ and $k$ some generic positive constants depending only on the given data of the problem but independent of the final time $T$, with $k$ being used in estimates from below. The values of $c$ and $k$ will be allowed to vary on occurrence, and will be assumed to be independent of any hypothetical regularization or approximation parameter. Specific values of $k$ and $c$ will be noted as $k_{i}, c_{i}, i \geq 1$.

\subsection{Energy estimate}

We start testing (1.1) with $\mathbf{u}$ to obtain

$$
\frac{1}{2} \frac{\mathrm{d}}{\mathrm{d} t}\|\mathbf{u}\|_{H_{\mathrm{div}}}^{2}+\|\nabla \mathbf{u}\|_{H_{\mathrm{div}}}^{2}-\int_{\Omega}(\nabla \varphi \otimes \nabla \varphi): \nabla \mathbf{u} \mathrm{d} x=0,
$$

where we also used the incompressibility constraint (1.2) and the periodic boundary conditions. Next, we test (1.4) with $\pi$. Using again periodicity and incompressibility we deduce

$$
\frac{\kappa}{2} \frac{\mathrm{d}}{\mathrm{d} t}\|\pi\|_{H}^{2}+\|\pi\|_{H}^{2}+\int_{\Omega} \nabla \pi \cdot \nabla \varphi \mathrm{d} x+\int_{\Omega} f(\varphi) \pi \mathrm{d} x=0
$$

Now we exploit the expression of $\pi(1.3)$ in order to compute the last two integrals in (4.2). Firstly we have

$$
\int_{\Omega} \nabla \pi \cdot \nabla \varphi \mathrm{d} x=\frac{1}{2} \frac{\mathrm{d}}{\mathrm{d} t}\|\nabla \varphi\|_{H}^{2}+\sigma\|\nabla \varphi\|_{H}^{2}+\epsilon\|\Delta \varphi\|_{H}^{2}+\int_{\Omega} \nabla \varphi \cdot \nabla(\mathbf{u} \cdot \nabla \varphi) \mathrm{d} x
$$

whereas the second term, thanks also to incompressibility, gives

$$
\int_{\Omega} f(\varphi) \pi \mathrm{d} x=\frac{\mathrm{d}}{\mathrm{d} t} \int_{\Omega} F(\varphi) \mathrm{d} x+\epsilon \int_{\Omega} f^{\prime}(\varphi)|\nabla \varphi|^{2} \mathrm{~d} x+\sigma \int_{\Omega} f(\varphi) \varphi \mathrm{d} x .
$$

Replacing (4.3)-(4.4) into (4.2), we deduce

$$
\begin{aligned}
& \frac{\mathrm{d}}{\mathrm{d} t}\left(\frac{\kappa}{2}\|\pi\|_{H}^{2}+\frac{1}{2}\|\nabla \varphi\|_{H}^{2}+\int_{\Omega} F(\varphi) \mathrm{d} x\right)+\|\pi\|_{H}^{2}+\sigma\|\nabla \varphi\|_{H}^{2}+\epsilon\|\Delta \varphi\|_{H}^{2} \\
& \quad+\sigma \int_{\Omega} f(\varphi) \varphi \mathrm{d} x+\int_{\Omega} \nabla \varphi \cdot \nabla(\mathbf{u} \cdot \nabla \varphi) \mathrm{d} x+\epsilon \int_{\Omega} f^{\prime}(\varphi)|\nabla \varphi|^{2} \mathrm{~d} x=0 .
\end{aligned}
$$

We now notice that, by standard integration by parts,

$$
\int_{\Omega} \nabla \varphi \cdot \nabla(\mathbf{u} \cdot \nabla \varphi) \mathrm{d} x=\int_{\Omega}(\nabla \varphi \otimes \nabla \varphi): \nabla \mathbf{u} \mathrm{d} x+\frac{1}{2} \int_{\Omega} \mathbf{u} \cdot \nabla|\nabla \varphi|^{2} \mathrm{~d} x,
$$

and the second summand on the right-hand side vanishes by incompressibility. Then, summing (4.1) together with (4.5) and taking (4.6) into account, we arrive at the energy estimate

$$
\begin{aligned}
\frac{\mathrm{d}}{\mathrm{d} t}( & \left.\frac{1}{2}\|\mathbf{u}\|_{H_{\mathrm{div}}}^{2}+\frac{\kappa}{2}\|\pi\|_{H}^{2}+\frac{1}{2}\|\nabla \varphi\|_{H}^{2}+\int_{\Omega} F(\varphi) \mathrm{d} x\right)+\|\nabla \mathbf{u}\|_{H_{\mathrm{div}}}^{2}+\|\pi\|_{H}^{2} \\
& +\sigma\|\nabla \varphi\|_{H}^{2}+\epsilon\|\Delta \varphi\|_{H}^{2}+\sigma \int_{\Omega} f(\varphi) \varphi \mathrm{d} x+\epsilon \int_{\Omega} f^{\prime}(\varphi)|\nabla \varphi|^{2} \mathrm{~d} x=0 .
\end{aligned}
$$


Now, since $F$ is just assumed to be $\lambda$-convex, in general the last term on the left-hand side may be nonpositive. In order to control it, we need to exploit the Gronwall inequality. Recalling (2.8) and Assumption (2.5) (and using in particular the $\lambda$-convexity of $F$ ), taking a generic $C>0$, we actually deduce

$$
\begin{aligned}
& \frac{\mathrm{d}}{\mathrm{d} t}\left[\|\mathbf{u}\|_{H_{\mathrm{div}}}^{2}+\kappa\|\pi\|_{H}^{2}+\|\nabla \varphi\|_{H}^{2}+\int_{\Omega} 2 F(\varphi) \mathrm{d} x+C\right]+2\|\nabla \mathbf{u}\|_{H_{\mathrm{div}}}^{2}+2\|\pi\|_{H}^{2} \\
& \quad+2(\sigma-\lambda \epsilon)\|\nabla \varphi\|_{H}^{2}+2 \epsilon\|\Delta \varphi\|_{H}^{2}+2 \sigma k_{p}\left(\|\varphi\|_{L^{p+2}(\Omega)}^{p+2}+\int_{\Omega} F(\varphi) \mathrm{d} x\right) \leq 2 \sigma c_{p}
\end{aligned}
$$

and the coefficient $2(\sigma-\lambda \epsilon)$ may be actually nonpositive (and it is always nonpositive when $\sigma=0$ and $\lambda>0)$.

Let us then note as $\mathcal{G}$ the sum of the terms in square brackets in (4.8). Now, recalling that $\mathbf{u}$ has zero spatial mean by (3.1) and (2.12), the Poincaré-Wirtinger inequality implies

$$
\|\nabla \mathbf{u}\|_{H_{\text {div }}}^{2} \geq k\|\mathbf{u}\|_{H_{\text {div }}}^{2}
$$

for some $k>0$. Hence, rearranging terms, (4.8) gives

$$
\begin{aligned}
\frac{\mathrm{d}}{\mathrm{d} t} \mathcal{G} & +\|\nabla \mathbf{u}\|_{H_{\mathrm{div}}}^{2}+k\|\mathbf{u}\|_{H_{\mathrm{div}}}^{2}+2\|\pi\|_{H}^{2}+\sigma k_{p}\|\nabla \varphi\|_{H}^{2}+2 \sigma k_{p} \int_{\Omega} F(\varphi) \mathrm{d} x \\
& +2 \epsilon\|\Delta \varphi\|_{H}^{2}+2 \sigma k_{p}\|\varphi\|_{L^{p+2}(\Omega)}^{p+2} \leq 2 \sigma c_{p}+\left(\sigma k_{p}-2 \sigma+2 \lambda \epsilon\right)\|\nabla \varphi\|_{H}^{2}
\end{aligned}
$$

Now, using (2.7) and (2.9), it is clear that $C$ can be chosen in such a way that

$$
\|\varphi\|_{V}^{2}+k^{\prime \prime}\|\varphi\|_{L^{p+2}(\Omega)}^{p+2} \leq\|\nabla \varphi\|_{H}^{2}+2 \int_{\Omega} F(\varphi) \mathrm{d} x+C \leq\|\varphi\|_{V}^{2}+2 K^{\prime \prime}\|\varphi\|_{L^{p+2}(\Omega)}^{p+2}+c .
$$

Hence, (4.10) implies the differential inequality

$$
\frac{\mathrm{d}}{\mathrm{d} t} \mathcal{G}+k \mathcal{G}+\|\nabla \mathbf{u}\|_{H_{\mathrm{div}}}^{2}+2 \epsilon\|\Delta \varphi\|_{H}^{2}+2 \sigma k_{p}\|\varphi\|_{L^{p+2}(\Omega)}^{p+2} \leq c\left(1+\|\nabla \varphi\|_{H}^{2}\right) .
$$

for some computable constants $k, c>0$ depending on the various parameters of the problem. Now, when $\sigma=0$ the right-hand side needs to be estimated by using Gronwall. Indeed, in that case (4.12), using (4.11), may be rewritten as

$$
\frac{\mathrm{d}}{\mathrm{d} t} \mathcal{G}+\|\nabla \mathbf{u}\|_{H_{\mathrm{div}}}^{2}+2 \epsilon\|\Delta \varphi\|_{H}^{2} \leq c_{1} \mathcal{G}+c_{2}
$$

for some constants $c_{1}, c_{2}>0$, whence Gronwall's inequality entails

$$
\mathcal{G}(t) \leq \mathcal{G}(0) e^{c_{1} t}+\frac{c_{2}}{c_{1}}
$$

as well as

$$
\int_{0}^{t}\left(\|\nabla \mathbf{u}(s)\|_{H_{\text {div }}}^{2}+2 \epsilon\|\Delta \varphi(s)\|_{H}^{2}\right) \mathrm{d} s \leq c\left(\mathcal{G}(0) e^{c_{1} t}+t\right) .
$$

On the other hand, when $\sigma>0$ it is clear that we can avoid exponential growth of the energy. Indeed, in that case the right-hand side of (4.12) can be controlled in the following way:

$$
c\|\nabla \varphi\|_{H_{\mathrm{div}}}^{2}=-c \int_{\Omega} \varphi \Delta \varphi \mathrm{d} x \leq \epsilon\|\Delta \varphi\|_{H}^{2}+\frac{1}{4 \epsilon}\|\varphi\|_{H}^{2} \leq \epsilon\|\Delta \varphi\|_{H}^{2}+\sigma k_{p}\|\varphi\|_{L^{p+2}}^{p+2}+c(\sigma, \epsilon, p),
$$

where in the last computation we have used (2.9). As a consequence, in place of (4.13) we get from (4.12) the better relation

$$
\frac{\mathrm{d}}{\mathrm{d} t} \mathcal{G}+k_{1} \mathcal{G}+\|\nabla \mathbf{u}\|_{H_{\mathrm{div}}}^{2}+\epsilon\|\Delta \varphi\|_{H}^{2}+\sigma k_{p}\|\varphi\|_{L^{p+2}(\Omega)}^{p+2} \leq c_{3},
$$

whence we immediately deduce

$$
\mathcal{G}(t) \leq \mathcal{G}(0) e^{-k_{1} t}+\frac{c_{3}}{k_{1}}
$$

and, integrating (4.17) over the generic interval $(t, t+1), t \geq 0$,

$$
\int_{t}^{t+1}\left(\|\nabla \mathbf{u}(s)\|_{H_{\mathrm{div}}}^{2}+\epsilon\|\Delta \varphi(s)\|_{H}^{2}+\sigma k_{p}\|\varphi(s)\|_{L^{p+2}(\Omega)}^{p+2}\right) \mathrm{d} s \leq \mathcal{G}(0) e^{-k_{1} t}+c_{3}+\frac{c_{3}}{k_{1}} .
$$




\subsection{Dissipative estimate for $\delta=\kappa$}

We start with testing (1.3) by $\pi$ to obtain

$$
\left(\pi, \varphi_{t}\right)=\|\pi\|_{H}^{2}-(\mathbf{u} \cdot \nabla \varphi, \pi)+\epsilon(\Delta \varphi, \pi)-\sigma(\varphi, \pi)
$$

Next, assuming $\delta=\kappa$, we test (1.4) by $\varphi$. Integrating by parts in time, we obtain

$$
\kappa\left(\pi, \varphi_{t}\right)=\kappa \frac{\mathrm{d}}{\mathrm{d} t}(\pi, \varphi)+(\pi, \varphi)+\kappa(\mathbf{u} \cdot \nabla \pi, \varphi)+\|\nabla \varphi\|_{H}^{2}+(f(\varphi), \varphi) .
$$

Combining the above relations, we deduce

$$
\begin{aligned}
\kappa \frac{\mathrm{d}}{\mathrm{d} t} & (\pi, \varphi)+(\pi, \varphi)+\|\nabla \varphi\|_{H}^{2}+(f(\varphi), \varphi) \\
& =\kappa\|\pi\|_{H}^{2}-\kappa[(\mathbf{u} \cdot \nabla \varphi, \pi)-(\mathbf{u} \cdot \nabla \pi, \varphi)]+\kappa \epsilon(\Delta \varphi, \pi)-\kappa \sigma(\varphi, \pi) .
\end{aligned}
$$

Now, we observe that, due to $\delta=\kappa$ and incompressibility, the transport terms actually vanish:

$$
(\mathbf{u} \cdot \nabla \varphi, \pi)+(\mathbf{u} \cdot \nabla \pi, \varphi)=\int_{\Omega} \mathbf{u} \cdot \nabla(\varphi \pi) \mathrm{d} x=-\int_{\Omega} \varphi \pi \operatorname{div} \mathbf{u} \mathrm{d} x=0 .
$$

Hence relation (4.22) reduces to

$$
\kappa \frac{\mathrm{d}}{\mathrm{d} t}(\pi, \varphi)+(\pi, \varphi)+\|\nabla \varphi\|_{H}^{2}+(f(\varphi), \varphi)=\kappa\|\pi\|_{H}^{2}+\kappa \epsilon(\Delta \varphi, \pi)-\kappa \sigma(\varphi, \pi) .
$$

Let us now multiply the above relation by $\eta>0$ and sum the result to (4.8). We get

$$
\begin{aligned}
& \frac{\mathrm{d}}{\mathrm{d} t}\left[\|\mathbf{u}\|_{H_{\mathrm{div}}}^{2}+\|\pi\|_{H}^{2}+\|\nabla \varphi\|_{H}^{2}+\int_{\Omega} 2 F(\varphi) \mathrm{d} x+C+\eta \kappa(\pi, \varphi)\right] \\
& \quad+2\|\nabla \mathbf{u}\|_{H_{\mathrm{div}}}^{2}+(2-\eta \kappa)\|\pi\|_{H}^{2}+2 \epsilon\|\Delta \varphi\|_{H}^{2}+\eta\|\nabla \varphi\|_{H}^{2}+\eta(f(\varphi), \varphi) \\
& \leq 2 \lambda \epsilon\|\nabla \varphi\|_{H}^{2}+\eta \kappa \epsilon(\Delta \varphi, \pi)-\eta(1+\kappa \sigma)(\pi, \varphi)
\end{aligned}
$$

and we need to control the terms on the right-hand side. First of all, we observe that

$$
\eta \kappa \epsilon(\Delta \varphi, \pi)-\eta(1+\kappa \sigma)(\pi, \varphi) \leq \frac{\eta \kappa^{2} \epsilon^{2}}{2}\|\Delta \varphi\|_{H}^{2}+\frac{\eta}{2}\|\varphi\|_{H}^{2}+\frac{\eta}{2}\left(1+(1+\kappa \sigma)^{2}\right)\|\pi\|_{H}^{2}
$$

On the other hand,

$$
2 \lambda \epsilon\|\nabla \varphi\|_{H}^{2}=2 \lambda \epsilon \int_{\Omega}(-\Delta \varphi) \varphi \mathrm{d} x \leq \frac{\epsilon}{2}\|\Delta \varphi\|_{H}^{2}+2 \lambda^{2} \epsilon\|\varphi\|_{H}^{2} .
$$

Replacing (4.25)-(4.26) into (4.24) we then deduce

$$
\begin{aligned}
& \frac{\mathrm{d}}{\mathrm{d} t}\left[\|\mathbf{u}\|_{H_{\mathrm{div}}}^{2}+\|\pi\|_{H}^{2}+\|\nabla \varphi\|_{H}^{2}+\int_{\Omega} 2 F(\varphi) \mathrm{d} x+C+\eta \kappa(\pi, \varphi)\right]+2\|\nabla \mathbf{u}\|_{H_{\mathrm{div}}}^{2} \\
& \quad+\left(2-\eta \kappa-\frac{\eta}{2}\left(1+(1+\kappa \sigma)^{2}\right)\right)\|\pi\|_{H}^{2}+\left(\frac{3 \epsilon}{2}-\frac{\eta \kappa^{2} \epsilon^{2}}{2}\right)\|\Delta \varphi\|_{H}^{2}+\eta\|\nabla \varphi\|_{H}^{2}+\eta(f(\varphi), \varphi) \\
& \quad \leq\left(\frac{\eta}{2}+2 \lambda^{2} \epsilon\right)\|\varphi\|_{H}^{2} .
\end{aligned}
$$

Now, we specify the choice of the "small" positive constant $\eta$. First, we need $\kappa \eta \leq 2 / 3$, in such a way that

$$
\begin{aligned}
& \kappa \eta(\pi, \varphi) \geq-\frac{1}{3}\|\pi\|_{H}^{2}-\frac{1}{3}\|\varphi\|_{H}^{2} \geq-\frac{1}{3}\|\pi\|_{H}^{2}-\int_{\Omega} F(\varphi) \mathrm{d} x-c, \\
& \kappa \eta(\pi, \varphi) \leq \frac{1}{3}\|\pi\|_{H}^{2}+\frac{1}{3}\|\varphi\|_{H}^{2}
\end{aligned}
$$


for some $c>0$, where also (2.9) has been used. Second, we need $\eta$ so small that the coefficients of the first two terms on the second row of (4.27) are strictly positive. We also note that, for such $\eta$, using $\mathcal{D}$ for the sum of the terms in square brackets in (4.27), there exists (a new value of) $C>0$ such that

$$
\begin{aligned}
& \|\nabla \varphi\|_{H}^{2}+\frac{2}{3}\|\pi\|_{H}^{2}+\|\mathbf{u}\|_{H_{\mathrm{div}}}^{2}+\int_{\Omega} F(\varphi) \mathrm{d} x \leq \mathcal{D} \\
& \quad \leq \frac{4}{3}\|\varphi\|_{V}^{2}+\frac{4}{3}\|\pi\|_{H}^{2}+\|\mathbf{u}\|_{H_{\mathrm{div}}}^{2}+\int_{\Omega} 2 F(\varphi) \mathrm{d} x+C .
\end{aligned}
$$

Next, thanks to (2.8) and (2.9), the right-hand side of (4.27) can be estimated as follows

$$
\left(\frac{\eta}{2}+2 \lambda^{2} \epsilon\right)\|\varphi\|_{H}^{2} \leq \frac{\eta}{2}(f(\varphi), \varphi)+c(\eta, \epsilon, \lambda)
$$

and the nonconstant term can be absorbed by the corresponding one on the left-hand side. Moreover, the term depending on $f$ on the left-hand side can be estimated from below by means of (2.8). Hence, using once more the Poincaré-Wirtinger inequality for $\mathbf{u}$, we see that (4.27) implies, for some $k_{2}, k_{3}>0$, the differential inequality

$$
\frac{\mathrm{d}}{\mathrm{d} t} \mathcal{D}+k_{2} \mathcal{D}+k_{3}\|\nabla \mathbf{u}\|_{H_{\mathrm{div}}}^{2}+k_{3} \epsilon\|\Delta \varphi\|_{H}^{2} \leq c_{4},
$$

with the constants $k_{2}, k_{3}, c_{4}>0$ depending on $\epsilon, \lambda$ and also through the choice of $\eta$. As a consequence, we deduce

$$
\mathcal{D}(t)+\int_{t}^{t+1} k_{3}\left(\|\nabla \mathbf{u}(s)\|_{H_{\mathrm{div}}}^{2}+\epsilon\|\Delta \varphi(s)\|_{H}^{2}\right) \mathrm{d} s \leq \mathcal{D}(0) e^{-k_{2} t}+\frac{c_{4}}{k_{2}}+c_{4}, \quad \forall t \geq 0 .
$$

\subsection{Proof of Theorem 1: existence of weak solutions}

In the sequel we consider a sequence $\left\{\left(\mathbf{u}_{n}, \varphi_{n}, \pi_{n}\right)\right\}, n \in \mathbb{N}$, of triplets satisfying system (1.1)-(1.4) in a suitable sense and complying, uniformly with respect to $n$, with the energy principle. More precisely, we assume that relation (4.12) is satisfied with constants $k, c$ independent of $n$. Hence, either (4.14)(4.15) or (4.18)-(4.19) also hold with constants independent of $n$. As a consequence, we can prove that any limit point (in a suitable sense) of a generic (nonrelabelled) subsequence of $\left\{\left(\mathbf{u}_{n}, \varphi_{n}, \pi_{n}\right)\right\}$ is a weak solution in the sense of Theorem 1. In Section 5 we will see how the present argument can be adapted to a Galerkin approximation scheme.

That said, we first observe that, from (4.14)-(4.15) (or from (4.18)-(4.19)), using the definition of $\mathcal{G}$ (cf. (4.8) ) and the structure property (4.11), we may deduce the following relations:

$$
\begin{array}{ll}
\mathbf{u}_{n} \rightarrow \mathbf{u} & \text { weakly star in } L^{\infty}\left(0, T ; H_{\text {div }}\right) \cap L^{2}\left(0, T ; V_{\text {div }}\right), \\
\varphi_{n} \rightarrow \varphi & \text { weakly star in } L^{\infty}(0, T ; V) \cap L^{\infty}\left(0, T ; L^{p+2}(\Omega)\right) \cap L^{2}\left(0, T ; H^{2}(\Omega)\right), \\
\pi_{n} \rightarrow \pi & \text { weakly star in } L^{\infty}(0, T ; H) .
\end{array}
$$

Here and below all the convergence properties will be intended to hold up to the extraction of (nonrelabelled) subsequences of $n \nearrow \infty$.

We now deduce some further consequence of the energy estimate that will be needed in order to take the limit of the product terms in the system. We start dealing with the velocity and observe that, for any $\mathbf{v} \in V_{\text {div }}$,

$$
\int_{\Omega}\left(\mathbf{u}_{n} \cdot \nabla \mathbf{u}_{n}\right) \cdot \mathbf{v} \mathrm{d} x=-\int_{\Omega}\left(\mathbf{u}_{n} \otimes \mathbf{u}_{n}\right): \nabla \mathbf{v} \mathrm{d} x \leq\left\|\mathbf{u}_{n}\right\|_{L^{4}(\Omega)}^{2}\|\nabla \mathbf{v}\|_{H} .
$$

Now, for $d=2$, thanks to Ladyzhenskaya's inequality, 4.35) implies

$$
\left\|\mathbf{u}_{n}\right\|_{L^{4}\left(0, T ; L^{4}(\Omega)\right)} \leq c,
$$

whence from (4.38) we obtain

$$
\left\|\mathbf{u}_{n} \cdot \nabla \mathbf{u}_{n}\right\|_{L^{2}\left(0, T ; V_{\text {div }}^{\prime}\right)} \leq c .
$$


On the other hand, for $d=3$, Sobolev's embeddings and interpolation only give

$$
\left\|\mathbf{u}_{n}\right\|_{L^{8 / 3}\left(0, T ; L^{4}(\Omega)\right)} \leq c,
$$

whence from (4.38) we deduce

$$
\left\|\mathbf{u}_{n} \cdot \nabla \mathbf{u}_{n}\right\|_{L^{4 / 3}\left(0, T ; V_{\text {div }}^{\prime}\right)} \leq c .
$$

Analogously, from (4.36) we have

$$
\begin{aligned}
& \left\|\nabla \varphi_{n}\right\|_{L^{4}\left(0, T ; L^{4}(\Omega)\right)} \leq c \quad \text { for } d=2, \\
& \left\|\nabla \varphi_{n}\right\|_{L^{8 / 3}\left(0, T ; L^{4}(\Omega)\right)} \leq c \quad \text { for } d=3,
\end{aligned}
$$

whence

$$
\begin{aligned}
& \left\|\nabla \varphi_{n} \otimes \nabla \varphi_{n}\right\|_{L^{2}(0, T ; H)} \leq c \quad \text { for } d=2, \\
& \left\|\nabla \varphi_{n} \otimes \nabla \varphi_{n}\right\|_{L^{4 / 3}(0, T ; H)} \leq c \quad \text { for } d=3 .
\end{aligned}
$$

Now, testing (1.1) (written for $\mathbf{u}_{n}, \varphi_{n}$ ) by $\mathbf{v} \in V_{\text {div }}$ and rearranging, we have

$$
\left\langle\mathbf{u}_{n, t}, \mathbf{v}\right\rangle=-\left\langle\nabla \mathbf{u}_{n}, \nabla \mathbf{v}\right\rangle+\left\langle\nabla \varphi_{n} \otimes \nabla \varphi_{n}, \nabla \mathbf{v}\right\rangle+\left\langle\mathbf{u}_{n} \otimes \mathbf{u}_{n}, \nabla \mathbf{v}\right\rangle,
$$

whence, using (4.40), (4.42) and (4.45)-(4.46), we easily infer

$$
\mathbf{u}_{n, t} \rightarrow \mathbf{u}_{t} \quad \text { weakly in } L^{q}\left(0, T ; V_{\text {div }}^{\prime}\right),
$$

where $q=2$ if $d=2$ and $q=4 / 3$ if $d=3$. Combining the above with (4.35) and applying the Aubin-Lions lemma, we then obtain

$$
\mathbf{u}_{n} \rightarrow \mathbf{u} \text { strongly in } C^{0}\left([0, T] ; V_{\text {div }}^{\prime}\right) \cap L^{2}\left(0, T ; H_{\text {div }}\right) .
$$

We now move to considering the behavior of $\varphi_{n}$. To this aim, we first notice that, using (4.39) and (4.43) in 2D, and using the first (4.35), the last (4.36) and Sobolev's embeddings in 3D, there follows

$$
\left\|\mathbf{u}_{n} \cdot \nabla \varphi_{n}\right\|_{L^{2}\left(0, T ; L^{s}(\Omega)\right)} \leq c,
$$

where $s=3 / 2$ if $d=3$ and $s=2$ if $d=2$. Hence, comparing terms in (1.3) and using again (4.35)-(4.37) it is not difficult to deduce

$$
\varphi_{n, t} \rightarrow \varphi_{t} \quad \text { weakly in } L^{2}\left(0, T ; L^{s}(\Omega)\right),
$$

with $s$ as above. Applying once more the Aubin-Lions lemma, we infer

$$
\varphi_{n} \rightarrow \varphi \text { strongly in } C^{0}([0, T] ; H) \cap L^{2}(0, T ; V) .
$$

As a consequence, we obtain (at least) that

$$
\nabla \varphi_{n} \otimes \nabla \varphi_{n} \rightarrow \nabla \varphi \otimes \nabla \varphi \quad \text { strongly in } L^{1}\left(0, T ; L^{1}(\Omega)\right) .
$$

This allows us to take the limit $n \nearrow \infty$ in relation (4.47) to get back (3.8). Moreover, it is clear that, from (4.49) and (4.52), there also follows (at least)

$$
\mathbf{u}_{n} \cdot \nabla \varphi_{n} \rightarrow \mathbf{u} \cdot \nabla \varphi \quad \text { strongly in } L^{1}\left(0, T ; L^{1}(\Omega)\right) .
$$

Actually, relations (4.53) and (4.54) could be improved, anyway they suffice for the sequel. Indeed, we can now write (1.3) at the $n$-level, take the limit $n \nearrow \infty$, and get back (3.9).

Finally, we need to pass to the limit in (1.4). Then, let us notice that (4.36) and (2.6) entail

$$
\left\|f\left(\varphi_{n}\right)\right\|_{L^{\infty}\left(0, T ; L^{\frac{p+2}{p+1}}(\Omega)\right)} \leq c .
$$


Combining this fact with the pointwise convergence resulting from (4.52) we then deduce

$$
f\left(\varphi_{n}\right) \rightarrow f(\varphi) \quad \text { weakly star in } L^{\infty}\left(0, T ; L^{\frac{p+2}{p+1}}(\Omega)\right) \text { and strongly in } L^{1}\left(0, T ; L^{1}(\Omega)\right) .
$$

Finally, if $\delta>0$, we need to take care of the convection term in (1.4). Combining (4.35) with (4.37) and using interpolation with the $2 \mathrm{D}$ embedding $H^{1}(\Omega) \hookrightarrow L^{q}(\Omega)$ for $1 \leq q<\infty$, we actually deduce that

$$
\left\|\mathbf{u}_{n} \pi_{n}\right\|_{L^{2}\left(0, T ; L^{r}(\Omega)\right)} \leq c_{r}
$$

where $r=3 / 2$ for $d=3$ and $r \in[1,2)$ for $d=2$. Then, using the second of (4.49), we infer

$$
\mathbf{u}_{n} \pi_{n} \rightarrow \mathbf{u} \pi \quad \text { weakly in } L^{2}\left(0, T ; L^{r}(\Omega)\right) .
$$

Hence, for any $v \in W^{1,3}(\Omega)$, using incompressibility and periodic boundary conditions, we deduce

$$
\int_{\Omega}\left(\mathbf{u}_{n} \cdot \nabla \pi_{n}\right) v \mathrm{~d} x=-\int_{\Omega} \pi_{n}\left(\mathbf{u}_{n} \cdot \nabla v\right) \mathrm{d} x \rightarrow-\int_{\Omega} \pi(\mathbf{u} \cdot \nabla v) \mathrm{d} x \quad \text { weakly in } L^{2}(0, T)
$$

and in particular the distributional divergence $\operatorname{div}(\pi \mathbf{u})$ lies in the dual space $L^{2}\left(0, T ;\left(W^{1,3}\right)^{\prime}(\Omega)\right)$.

Using (4.35)-(4.37) together with (4.55) and (4.57), comparing terms in the $n$-version of (1.4), and using continuity of the embedding $L^{\infty}\left(0, T ; L^{\frac{p+2}{p+1}}(\Omega)\right) \subset L^{2}\left(0, T ;\left(W^{1,3}\right)^{\prime}(\Omega)\right)$, we then obtain

$$
\pi_{n, t} \rightarrow \pi_{t} \quad \text { weakly in } L^{2}\left(0, T ;\left(W^{1,3}\right)^{\prime}(\Omega)\right) .
$$

This fact, by the Aubin-Lions lemma and (4.37), also yields the strong convergence

$$
\pi_{n} \rightarrow \pi \text { strongly in } C^{0}\left([0, T] ; V^{\prime}\right) .
$$

The above relations permit us to pass to the limit in (1.4), which may be reinterpreted as an equality in $L^{2}\left(0, T ;\left(W^{1,3}\right)^{\prime}(\Omega)\right.$ ) (or also as an equality in $\left(W^{1,3}\right)^{\prime}(\Omega)$, almost everywhere in time). Namely, in the limit we get back exactly (3.10). Note also that the regularity conditions (3.2)-(3.6) in the statement of Theorem 1 are a direct consequence of (4.35)-(4.37), (4.48) (4.51) and (4.60).

Finally, we observe that the strong convergence relations (4.49), (4.52), (4.61) imply

$$
\left.\left.\left(\mathbf{u}_{n}, \varphi_{n}, \pi_{n}\right)\right|_{t=0} \rightarrow(\mathbf{u}, \varphi, \pi)\right|_{t=0} \text { strongly in } V_{\text {div }}^{\prime} \times H \times V^{\prime} .
$$

Hence, assuming that the triplet $\left(\mathbf{u}_{n}, \varphi_{n}, \pi_{n}\right)$ satisfies an initial condition of the form

$$
\left.\left(\mathbf{u}_{n}, \varphi_{n}, \pi_{n}\right)\right|_{t=0}=\left(\mathbf{u}_{0, n}, \varphi_{0, n}, \pi_{0, n}\right),
$$

where $\left(\mathbf{u}_{0, n}, \varphi_{0, n}, \pi_{0, n}\right)$ tends to $\left(\mathbf{u}_{0}, \varphi_{0}, \pi_{0}\right)$ in a suitable way, letting $n \nearrow \infty$, we obtain (3.11) in the limit, which concludes the proof of Theorem [1

Remark 5. It is worth discussing a bit more the occurrence of the viscous regularization term in (1.3) at the light of the a priori estimates. Actually, if that term is omitted (i.e., if $\epsilon=0$ ), the latter convergence in (4.36) would be lacking and we would not have any $L^{p}$-information on second space derivatives of $\varphi$. Hence, the $L^{2}(0, T ; V)$-convergence in (4.52) would also be missing and we could not take the limit of the right-hand side of (1.1) as specified in (4.53).

\subsection{Proof of Proposition 2: dissipativity}

In the case $\sigma>0$ we can take advantage of estimate (4.18), whereas for $\delta=\kappa$ we have relation (4.34). Of course, these bounds hold a priori for the approximating solutions $\left(\mathbf{u}_{n}, \varphi_{n}, \pi_{n}\right)$. On the other hand, they pass to the limit $n \nearrow \infty$ because the quantities on the right-hand sides are independent of $n$ and we can use semicontinuity of norms with respect to weak or weak star convergence when we take the limit. Hence we obtain that (4.18), or (4.34), also holds for limit solution(s) $(\mathbf{u}, \varphi, \pi)$. Noting that the functionals $\mathcal{G}$ (cf. (4.8)) and $\mathcal{D}$ (cf. (4.27)) control both from above and from below the norms specified in the statement, it is then apparent that (4.18) (or (4.34)) implies the desired bound (3.13) for every $t \geq T_{0}$, with $T_{0}$ depending only on the magnitude of the initial energy, i.e., on the norms in (3.12). 
Remark 6. One may wonder if the dissipative estimate (3.13) (possibly combined with the regularity estimates obtained in the proof of existence of strong solutions) could be used to prove existence of the global attractor at least in the $2 \mathrm{D}$ case. We do not address this interesting issue here, but we limit ourselves to observe that the problem seems nontrivial and its resolution may require the use of some careful decomposition method. Indeed, while the variables $\mathbf{u}$ and $\varphi$ enjoy some regularization property (if they are, respectively, in $H_{\text {div }}$ and in $V$ at the initial time, then they are in $V_{\text {div }}$ and in $H^{2}(\Omega)$ for some small $t>0$ ), i.e., they have a parabolic behavior, on the other hand the variable $\pi$ has a hyperbolic behavior, i.e. it does not seem to regularize in time (compare (3.5) for $\pi$ with (3.2) and (3.3) for $\mathbf{u}$ and $\varphi$ ).

\subsection{Proof of Theorem 3: existence of strong solutions}

The proof is based on some additional regularity estimates. As before, we work directly on system (1.1)-(1.4). We postpone to the next section the justification of this argument at the light of the approximation scheme.

First of all, we multiply (1.1) with $-\Delta \mathbf{u}$ and integrate over $\Omega$ to obtain

$$
\begin{aligned}
0 & =\int_{\Omega}\left(-\mathbf{u}_{t} \Delta \mathbf{u}+|\Delta \mathbf{u}|^{2}-\operatorname{div}(\nabla \varphi \otimes \nabla \varphi) \Delta \mathbf{u}-\nabla p \Delta \mathbf{u}-\operatorname{div}(\mathbf{u} \otimes \mathbf{u}) \Delta \mathbf{u}\right) \mathrm{d} x \\
& =\frac{1}{2} \frac{\mathrm{d}}{\mathrm{d} t}\|\nabla \mathbf{u}\|_{H_{\mathrm{div}}}^{2}+\|\Delta \mathbf{u}\|_{H_{\mathrm{div}}}^{2}+\int_{\Omega}\left(-\left(D^{2} \varphi \nabla \varphi\right) \cdot \Delta \mathbf{u}-\Delta \varphi(\nabla \varphi \cdot \Delta \mathbf{u})\right) \mathrm{d} x .
\end{aligned}
$$

Indeed, the convection term on the first row vanishes due to $d=2$ (see, e.g., [33, p. 242]).

Now using Ladyzhenskaya's and Young's inequalities with 2D Sobolev embeddings, we estimate the last two terms as follows:

$$
\begin{aligned}
& \left|\int_{\Omega}\left(-\left(D^{2} \varphi \nabla \varphi\right) \cdot \Delta \mathbf{u}-\Delta \varphi(\nabla \varphi \cdot \Delta \mathbf{u})\right) \mathrm{d} x\right| \\
& \quad \leq\|\nabla \varphi\|_{L^{4}(\Omega)}\left\|D^{2} \varphi\right\|_{L^{4}(\Omega)}\|\Delta \mathbf{u}\|_{H_{\mathrm{div}}} \\
& \quad \leq\|\nabla \varphi\|_{H}^{1 / 2}\|\nabla \varphi\|_{V}^{1 / 2}\left\|D^{2} \varphi\right\|_{H}^{1 / 2}\left\|D^{2} \varphi\right\|_{V}^{1 / 2}\|\Delta \mathbf{u}\|_{H_{\mathrm{div}}} \\
& \quad \leq \alpha\|\Delta \mathbf{u}\|_{H_{\mathrm{div}}}^{2}+c_{\alpha}\|\nabla \varphi\|_{V}\left\|D^{2} \varphi\right\|_{H}\left\|D^{3} \varphi\right\|_{H} \\
& \quad \leq \alpha\|\Delta \mathbf{u}\|_{H_{\mathrm{div}}}^{2}+\alpha\left\|D^{3} \varphi\right\|_{H}^{2}+c_{\alpha}\left\|D^{2} \varphi\right\|_{H}^{4} \\
& \quad \leq \alpha\|\Delta \mathbf{u}\|_{H_{\mathrm{div}}}^{2}+\alpha\|\nabla \Delta \varphi\|_{H}^{2}+c_{\alpha}\|\Delta \varphi\|_{H}^{4},
\end{aligned}
$$

where $\alpha>0$ denotes small constants to be chosen later and the constants $c_{\alpha}>0$ are correspondingly large. In the above computation we used the uniform in time $V$-bound for $\varphi$ resulting from the energy bound (cf. (4.36) ) and elliptic regularity.

Next, we deal with the Allen-Cahn system. Actually, testing (1.3) with $\Delta^{2} \varphi$, (1.4) with $-\Delta \pi$, summing the results, and performing standard manipulations (note in particular that a couple of terms cancels out), we obtain

$$
\begin{aligned}
\frac{1}{2} \frac{\mathrm{d}}{\mathrm{d} t}\left[\kappa\|\nabla \pi\|_{H}^{2}+\|\Delta \varphi\|_{H}^{2}\right]+\|\nabla \pi\|_{H}^{2}+\sigma\|\Delta \varphi\|_{H}^{2}+\epsilon\|\nabla \Delta \varphi\|_{H}^{2} \\
\quad=-\int_{\Omega} f^{\prime}(\varphi) \nabla \varphi \nabla \pi \mathrm{d} x+\int_{\Omega}(\nabla \mathbf{u} \nabla \varphi) \cdot \nabla \Delta \varphi \mathrm{d} x+\int_{\Omega}\left(D^{2} \varphi \mathbf{u}\right) \cdot \nabla \Delta \varphi \mathrm{d} x=: \sum_{j=1}^{3} I_{j}
\end{aligned}
$$

and we need to control the terms $I_{j}$ on the right-hand side. We actually have

$$
\begin{aligned}
\left|I_{1}\right| & \leq c \int_{\Omega}\left(1+|\varphi|^{p}\right)|\nabla \varphi \| \nabla \pi| \mathrm{d} x \\
& \leq c\left\||\varphi|^{p}\right\|_{L^{4}(\Omega)}\|\nabla \varphi\|_{L^{4}(\Omega)}\|\nabla \pi\|_{H}+c\|\nabla \varphi\|_{H}\|\nabla \pi\|_{H} \\
& \leq c\left(1+\|\varphi\|_{L^{4 p}(\Omega)}^{p}\|\nabla \varphi\|_{H}^{1 / 2}\|\nabla \varphi\|_{V}^{1 / 2}\right)\|\nabla \pi\|_{H} \\
& \leq c\left(1+\|\varphi\|_{H^{2}(\Omega)}^{1 / 2}\right)\|\nabla \pi\|_{H} \\
& \leq \alpha\|\nabla \pi\|_{H}^{2}+c_{\alpha}\left(1+\|\Delta \varphi\|_{H}\right),
\end{aligned}
$$




$$
\begin{aligned}
\left|I_{2}\right| \leq\|\nabla \mathbf{u}\|_{H_{\text {div }}}^{1 / 2}\|\nabla \mathbf{u}\|_{V_{\text {div }}}^{1 / 2}\|\nabla \varphi\|_{H}^{1 / 2}\|\nabla \varphi\|_{V}^{1 / 2}\|\nabla \Delta \varphi\|_{H} \\
\leq \alpha\|\nabla \mathbf{u}\|_{V_{\text {div }}}^{2}+\alpha\|\nabla \Delta \varphi\|_{H}^{2}+c_{\alpha}\|\nabla \varphi\|_{H}^{2}\|\nabla \varphi\|_{V}^{2}\|\nabla \mathbf{u}\|_{H_{\text {div }}}^{2} \\
\leq \alpha\|\Delta \mathbf{u}\|_{H_{\text {div }}}^{2}+\alpha\|\nabla \Delta \varphi\|_{H}^{2}+c_{\alpha}\|\Delta \varphi\|_{H}^{2}\|\nabla \mathbf{u}\|_{H_{\text {div }}}^{2}, \\
\left|I_{3}\right| \leq\|\mathbf{u}\|_{L^{4}(\Omega)}\left\|D^{2} \varphi\right\|_{L^{4}(\Omega)}\|\nabla \Delta \varphi\|_{H} \\
\quad \leq c\|\mathbf{u}\|_{H_{\text {div }}}^{1 / 2}\|\mathbf{u}\|_{V_{\text {div }}}^{1 / 2}\left\|D^{2} \varphi\right\|_{H}^{1 / 2}\left\|D^{3} \varphi\right\|_{H}^{1 / 2}\|\nabla \Delta \varphi\|_{H} \\
\quad \leq c\|\mathbf{u}\|_{V_{\text {div }} / 2}^{1 / 2}\|\varphi \varphi\|_{H}^{1 / 2}\|\nabla \Delta \varphi\|_{H}^{3 / 2} \\
\quad \leq \alpha\|\nabla \Delta \varphi\|_{H}^{2}+c_{\alpha}\|\nabla \mathbf{u}\|_{H_{\text {div }}}^{2}\|\Delta \varphi\|_{H}^{2},
\end{aligned}
$$

where $\alpha$ and $c_{\alpha}$ are as above (of course their value can vary on occurrence) and we have repeatedly used assumption (2.5), 2D-Sobolev embeddings, Young's and Ladyzhenskaya's inequalities, and the information already obtained with the energy bound (cf. (4.35)-(4.37)).

Now we sum (4.64) to (4.66). Using (4.65), (4.67), (4.68) and (4.69), we then deduce

$$
\begin{gathered}
\frac{1}{2} \frac{\mathrm{d}}{\mathrm{d} t}\left[\|\nabla \mathbf{u}\|_{H_{\mathrm{div}}}^{2}+\kappa\|\nabla \pi\|_{H}^{2}+\|\Delta \varphi\|_{H}^{2}\right]+\|\Delta \mathbf{u}\|_{H_{\mathrm{div}}}^{2}+\|\nabla \pi\|_{H}^{2}+\sigma\|\Delta \varphi\|_{H}^{2}+\epsilon\|\nabla \Delta \varphi\|_{H}^{2} \\
\quad \leq \alpha\|\Delta \mathbf{u}\|_{H_{\mathrm{div}}}^{2}+\alpha\|\nabla \Delta \varphi\|_{H}^{2}+c_{\alpha}+c_{\alpha}\|\Delta \varphi\|_{H}^{4}+\alpha\|\nabla \pi\|_{H}^{2}+c_{\alpha}\|\Delta \varphi\|_{H}^{2}\|\nabla \mathbf{u}\|_{H_{\mathrm{div}}}^{2} .
\end{gathered}
$$

Now, we can take the various $\alpha$ 's small enough (in a way that also depends on $\epsilon$ ) so that the above reduces to

$$
\begin{aligned}
& \frac{\mathrm{d}}{\mathrm{d} t}\left[\|\nabla \mathbf{u}\|_{H_{\mathrm{div}}}^{2}+\kappa\|\nabla \pi\|_{H}^{2}+\|\Delta \varphi\|_{H}^{2}\right]+\|\Delta \mathbf{u}\|_{H_{\mathrm{div}}}^{2}+\|\nabla \pi\|_{H}^{2}+\sigma\|\Delta \varphi\|_{H}^{2}+\epsilon\|\nabla \Delta \varphi\|_{H}^{2} \\
& \quad \leq c\left(1+\|\Delta \varphi\|_{H}^{2}\right)\left(1+\|\Delta \varphi\|_{H}^{2}+\|\nabla \mathbf{u}\|_{H_{\mathrm{div}}}^{2}\right) .
\end{aligned}
$$

This inequality has the structure

$$
\frac{\mathrm{d}}{\mathrm{d} t} \mathcal{E}_{1}(t)+\mathcal{D}_{1}(t) \leq m(t)\left(1+\mathcal{E}_{1}(t)\right)
$$

where $\mathcal{E}_{1}$ denotes the quantity in square brackets on the left-hand side, $\mathcal{D}_{1}$ is the sum of the remaining terms, and the function

$$
t \mapsto m(t):=c\left(1+\|\Delta \varphi\|_{H}^{2}\right)
$$

belongs to $L^{1}(0, T)$ thanks to the $L^{2}\left(0, T ; H^{2}(\Omega)\right)$-bound for $\varphi$ following from the energy estimate (cf. (4.36) ).

Applying Gronwall's Lemma to the function $t \mapsto \mathcal{E}_{1}(t)$ we then conclude that

$$
\begin{aligned}
& \mathbf{u} \in L^{\infty}\left(0, T ; V_{\mathrm{div}}\right), \\
& \pi \in L^{\infty}(0, T ; V), \\
& \varphi \in L^{\infty}\left(0, T ; H^{2}(\Omega)\right) .
\end{aligned}
$$

Note that the improved regularity assumptions on the initial data (3.14) have also been used here.

Then, integrating once more (4.72) in time we also deduce

$$
\begin{aligned}
& \mathbf{u} \in L^{2}\left(0, T ; H^{2}(\Omega)\right), \\
& \nabla \Delta \varphi \in L^{2}(0, T ; H),
\end{aligned}
$$

whence, using once more elliptic regularity we infer

$$
\varphi \in L^{2}\left(0, T ; H^{3}(\Omega)\right) .
$$

Finally, comparing terms in the equations of the system and using the above bounds, standard manipulations permit us to deduce also

$$
\begin{aligned}
& \mathbf{u}_{t} \in L^{2}\left(0, T ; H_{\text {div }}\right), \\
& \varphi_{t} \in L^{2}(0, T ; V), \\
& \pi_{t} \in L^{2}(0, T ; H),
\end{aligned}
$$


which is the last bound we need.

Now, in order to complete the proof, we interpret the information obtained above in the framework of an approximation scheme. In this respect, (4.74), (4.75), (4.76), and (4.77) can be seen as a priori estimates uniform with respect to the approximation parameter $n$. Consequently, the convergence relations (4.35)-(4.37) may also be improved in the corresponding way. Finally, we may observe that, in view of the enhanced regularity, all terms in equations (1.1) and (1.4) lie in some $L^{p}$-space and may be consequently interpreted in the pointwise sense, as noted in the statement of the theorem. This completes the proof of Theorem 3

\subsection{Proof of Theorem 4: uniqueness}

Let us given a pair of strong solutions $\left(\mathbf{u}_{1}, \varphi_{1}, \pi_{1}\right)$ and $\left(\mathbf{u}_{2}, \varphi_{2}, \pi_{2}\right)$ of (1.1)-(1.4) emanating from the same initial datum $\left(\mathbf{u}_{0}, \varphi_{0}, \pi_{0}\right)$ satisfying (3.14). We will show that the two solutions do coincide. We perform the proof in the more difficult case $d=3$ (as said, in this situation the result is conditional because strong solutions are not known to exist); of course the argument extends to $d=2$ where we actually have better embeddings. That said, we put $\mathbf{u}:=\mathbf{u}_{1}-\mathbf{u}_{2}, \varphi:=\varphi_{1}-\varphi_{2}, \pi:=\pi_{1}-\pi_{2}$ and $p:=p_{1}-p_{2}$. Then, writing (1.1) for the two solutions, and taking the difference, we get

$$
\mathbf{u}_{t}-\Delta \mathbf{u}+\operatorname{div}\left(\mathbf{u} \otimes \mathbf{u}_{1}\right)+\operatorname{div}\left(\mathbf{u}_{2} \otimes \mathbf{u}\right)+\nabla p+\operatorname{div}\left(\nabla \varphi \otimes \nabla \varphi_{1}\right)+\operatorname{div}\left(\nabla \varphi_{2} \otimes \nabla \varphi\right)=0 .
$$

Proceeding in the same way for (1.3) and (1.4) we obtain

$$
\begin{aligned}
& \pi=\varphi_{t}+\mathbf{u}_{1} \cdot \nabla \varphi+\mathbf{u} \cdot \nabla \varphi_{2}-\epsilon \Delta \varphi+\sigma \varphi \\
& \kappa \pi_{t}+\pi+\delta \mathbf{u}_{1} \cdot \nabla \pi+\delta \mathbf{u} \cdot \nabla \pi_{2}-\Delta \varphi+f\left(\varphi_{1}\right)-f\left(\varphi_{2}\right)=0 .
\end{aligned}
$$

We now work on system (4.78)-(4.80) with the aim of getting a contractive estimate. We start testing (4.78) with $\mathbf{u}$ to get

$$
\begin{gathered}
\frac{1}{2} \frac{\mathrm{d}}{\mathrm{d} t}\|\mathbf{u}\|_{H_{\mathrm{div}}}^{2}+\|\nabla \mathbf{u}\|_{H_{\mathrm{div}}}^{2}=\int_{\Omega}\left(\left(\nabla \varphi \otimes \nabla \varphi_{1}\right)+\left(\nabla \varphi_{2} \otimes \nabla \varphi\right)\right): \nabla \mathbf{u} \mathrm{d} x \\
+\int_{\Omega}\left(\left(\mathbf{u} \otimes \mathbf{u}_{1}\right)+\left(\mathbf{u}_{2} \otimes \mathbf{u}\right)\right): \nabla \mathbf{u} \mathrm{d} x=: K_{1}+K_{2} .
\end{gathered}
$$

Let us now provide a bound of the terms $K_{i}$ on the right-hand side. First, we have

$$
\begin{aligned}
\left|K_{1}\right| & \leq\|\nabla \mathbf{u}\|_{H_{\mathrm{div}}}\|\nabla \varphi\|_{L^{3}(\Omega)}\left(\left\|\nabla \varphi_{1}\right\|_{L^{6}(\Omega)}+\left\|\nabla \varphi_{2}\right\|_{L^{6}(\Omega)}\right) \\
& \leq c\|\nabla \mathbf{u}\|_{H_{\mathrm{div}}}\|\nabla \varphi\|_{H}^{1 / 2}\|\Delta \varphi\|_{H}^{1 / 2} \leq \frac{1}{8}\|\nabla \mathbf{u}\|_{H_{\mathrm{div}}}^{2}+\frac{\epsilon}{6}\|\Delta \varphi\|_{H}^{2}+c_{\epsilon}\|\nabla \varphi\|_{H}^{2},
\end{aligned}
$$

where we have used Sobolev's embeddings, Young's inequality, regularity (3.16) both for $\varphi_{1}$ and for $\varphi_{2}$, and elliptic regularity results (in order to control the $V$-norm of $\nabla \varphi$ with the $H$-norm of $\Delta \varphi$ ). Secondly, we have

$$
\begin{aligned}
\left|K_{2}\right| & \leq\|\nabla \mathbf{u}\|_{H_{\text {div }}}\|\mathbf{u}\|_{L^{3}(\Omega)}\left(\left\|\mathbf{u}_{1}\right\|_{L^{6}(\Omega)}+\left\|\mathbf{u}_{2}\right\|_{L^{6}(\Omega)}\right) \\
& \leq c\|\nabla \mathbf{u}\|_{H_{\text {div }}}^{3 / 2}\|\mathbf{u}\|_{H_{\text {div }}}^{1 / 2} \leq \frac{1}{8}\|\nabla \mathbf{u}\|_{H_{\text {div }}}^{2}+c\|\mathbf{u}\|_{H_{\text {div }}}^{2},
\end{aligned}
$$

having used regularity (3.15) both for $\mathbf{u}_{1}$ and for $\mathbf{u}_{2}$, Sobolev's embeddings, and the Poincaré-Wirtinger inequality (recall that both $\mathbf{u}_{1}$ and $\mathbf{u}_{2}$ have zero spatial mean).

Next, we multiply (4.79) by $-\Delta \varphi$ to deduce

$$
\frac{1}{2} \frac{\mathrm{d}}{\mathrm{d} t}\|\nabla \varphi\|_{H}+\epsilon\|\Delta \varphi\|_{H}^{2}+\sigma\|\nabla \varphi\|_{H}^{2}=-(\pi, \Delta \varphi)+\left(\mathbf{u}_{1} \cdot \nabla \varphi+\mathbf{u} \cdot \nabla \varphi_{2}, \Delta \varphi\right) .
$$

Correspondingly, we multiply (4.80) by $\pi$. Standard manipulations give

$$
\frac{\kappa}{2} \frac{\mathrm{d}}{\mathrm{d} t}\|\pi\|_{H}+\|\pi\|_{H}^{2}=-\delta\left(\mathbf{u}_{1} \cdot \nabla \pi+\mathbf{u} \cdot \nabla \pi_{2}, \pi\right)+(\pi, \Delta \varphi)-\left(f\left(\varphi_{1}\right)-f\left(\varphi_{2}\right), \pi\right) .
$$


Combining the previous two relations we obtain

$$
\begin{aligned}
\frac{1}{2} \frac{\mathrm{d}}{\mathrm{d} t} & \|\nabla \varphi\|_{H}+\frac{\kappa}{2} \frac{\mathrm{d}}{\mathrm{d} t}\|\pi\|_{H}+\epsilon\|\Delta \varphi\|_{H}^{2}+\sigma\|\nabla \varphi\|_{H}^{2}+\|\pi\|_{H}^{2} \\
& =\left(\mathbf{u}_{1} \cdot \nabla \varphi, \Delta \varphi\right)+\left(\mathbf{u} \cdot \nabla \varphi_{2}, \Delta \varphi\right)-\delta\left(\mathbf{u}_{1} \cdot \nabla \pi, \pi\right)-\delta\left(\mathbf{u} \cdot \nabla \pi_{2}, \pi\right)-\left(f\left(\varphi_{1}\right)-f\left(\varphi_{2}\right), \pi\right) \\
& =: J_{1}+J_{2}+J_{3}+J_{4}+J_{5},
\end{aligned}
$$

and we need to control the quantities $J_{i}$ on the right-hand side. First of all,

$$
\begin{aligned}
\left|J_{1}\right| & \leq\left\|\mathbf{u}_{1}\right\|_{L^{6}(\Omega)}\|\nabla \varphi\|_{L^{3}(\Omega)}\|\Delta \varphi\|_{H} \\
& \leq\left\|\mathbf{u}_{1}\right\|_{V_{\text {div }}}\|\nabla \varphi\|_{H}^{1 / 2}\|\Delta \varphi\|_{H}^{3 / 2} \leq \frac{\epsilon}{6}\|\Delta \varphi\|_{H}^{2}+c_{\epsilon}\|\nabla \varphi\|_{H}^{2},
\end{aligned}
$$

where we used interpolation, regularity (3.15) for $\mathbf{u}_{1}$, and elliptic regularity results for $\varphi$. Next, using the fact that $\mathbf{u}$ has zero spatial mean and the regularity (3.16) for $\varphi_{2}$, we obtain

$$
\begin{aligned}
\left|J_{2}\right| & \leq\|\mathbf{u}\|_{L^{3}(\Omega)}\left\|\nabla \varphi_{2}\right\|_{L^{6}(\Omega)}\|\Delta \varphi\|_{H} \\
& \leq\|\mathbf{u}\|_{H_{\mathrm{div}}}^{1 / 2}\|\nabla \mathbf{u}\|_{H_{\mathrm{div}}}^{1 / 2}\left\|\varphi_{2}\right\|_{H^{2}(\Omega)}\|\Delta \varphi\|_{H} \leq \frac{1}{8}\|\nabla \mathbf{u}\|_{H_{\mathrm{div}}}^{2}+\frac{\epsilon}{6}\|\Delta \varphi\|_{H}^{2}+c_{\epsilon}\|\mathbf{u}\|_{H_{\mathrm{div}}}^{2} .
\end{aligned}
$$

On the other hand, by incompressibility,

$$
J_{3}=-\frac{\delta}{2} \int_{\Omega} \mathbf{u}_{1} \cdot \nabla \pi^{2} \mathrm{~d} x=0,
$$

whereas the subsequent term, for $\delta>0$, can be controlled only in case we have the additional regularity (3.22) (here for $i=2$ ):

$$
\begin{aligned}
\left|J_{4}\right| & \leq \delta\|\mathbf{u}\|_{L^{6}(\Omega)}\left\|\nabla \pi_{2}\right\|_{L^{3}(\Omega)}\|\pi\|_{H} \\
& \leq \frac{1}{8}\|\nabla \mathbf{u}\|_{H_{\mathrm{div}}}^{2}+c \delta^{2}\left\|\nabla \pi_{2}\right\|_{L^{3}(\Omega)}^{2}\|\pi\|_{H}^{2} .
\end{aligned}
$$

Finally, using (2.5), we have

$$
\left|J_{5}\right| \leq c \int_{\Omega}\left(1+\left|\varphi_{2}\right|^{p}+\left|\varphi_{1}\right|^{p}\right)|\pi||\varphi| \mathrm{d} x \leq c\|\varphi\|_{H}^{2}+c\|\pi\|_{H}^{2} .
$$

Now, we can take the sum of (4.81) and (4.86). Using (4.82)-(4.83) and (4.87)-(4.91), we then deduce

$$
\begin{gathered}
\frac{1}{2} \frac{\mathrm{d}}{\mathrm{d} t}\|\mathbf{u}\|_{H_{\mathrm{div}}}^{2}+\frac{1}{2}\|\nabla \mathbf{u}\|_{H_{\mathrm{div}}}^{2}+\frac{1}{2} \frac{\mathrm{d}}{\mathrm{d} t}\|\nabla \varphi\|_{H}+\frac{\kappa}{2} \frac{\mathrm{d}}{\mathrm{d} t}\|\pi\|_{H}+\frac{\epsilon}{2}\|\Delta \varphi\|_{H}^{2}+\sigma\|\nabla \varphi\|_{H}^{2} \\
\leq c_{\epsilon}\|\nabla \varphi\|_{H}^{2}+c_{\epsilon}\|\mathbf{u}\|_{H_{\mathrm{div}}}^{2}+c\left(1+\delta^{2}\left\|\nabla \pi_{2}\right\|_{L^{3}(\Omega)}^{2}\right)\|\pi\|_{H}^{2}+c\|\varphi\|_{H}^{2} .
\end{gathered}
$$

To control the last term on the right-hand side, we need to test (4.79) with $\varphi$. This procedure yields

$$
\begin{aligned}
& \frac{1}{2} \frac{\mathrm{d}}{\mathrm{d} t}\|\varphi\|_{H}^{2}+\epsilon\|\nabla \varphi\|_{H}^{2}+\sigma\|\varphi\|_{H}^{2} \\
& =\int_{\Omega} \pi \varphi \mathrm{d} x-\int_{\Omega}\left(\mathbf{u}_{1} \cdot \nabla \varphi\right) \varphi \mathrm{d} x-\int_{\Omega}\left(\mathbf{u} \cdot \nabla \varphi_{2}\right) \varphi \mathrm{d} x=: H_{1}+H_{2}+H_{3} .
\end{aligned}
$$

Now, $\mathrm{H}_{2}$ is readily seen to be 0 thanks to incompressibility. On the other hand, it is easy to check that

$$
\begin{aligned}
& \left|H_{1}\right| \leq c\|\varphi\|_{H}^{2}+c\|\pi\|_{H}^{2}, \\
& \left|H_{3}\right| \leq\|\mathbf{u}\|_{L^{4}(\Omega)}\left\|\nabla \varphi_{2}\right\|_{L^{4}(\Omega)}\|\varphi\|_{H} \leq \frac{1}{4}\|\nabla \mathbf{u}\|_{H_{\text {div }}}^{2}+c\|\varphi\|_{H^{\prime}}^{2} .
\end{aligned}
$$


Hence, summing (4.93) to (4.92), neglecting some positive quantities on the left-hand side, and taking (4.94)- (4.95) into account, we finally arrive at

$$
\begin{gathered}
\frac{1}{2} \frac{\mathrm{d}}{\mathrm{d} t}\|\mathbf{u}\|_{H_{\mathrm{div}}}^{2}+\frac{1}{4}\|\nabla \mathbf{u}\|_{H_{\mathrm{div}}}^{2}+\frac{1}{2} \frac{\mathrm{d}}{\mathrm{d} t}\|\varphi\|_{V}^{2}+\frac{\kappa}{2} \frac{\mathrm{d}}{\mathrm{d} t}\|\pi\|_{H}^{2}+\frac{\epsilon}{2}\|\Delta \varphi\|_{H}^{2}+\sigma\|\nabla \varphi\|_{H}^{2} \\
\leq c_{\epsilon}\|\nabla \varphi\|_{H}^{2}+c_{\epsilon}\|\mathbf{u}\|_{H_{\mathrm{div}}}^{2}+c\left(1+\delta^{2}\left\|\nabla \pi_{2}\right\|_{L^{3}(\Omega)}^{2}\right)\|\pi\|_{H}^{2}+c\|\varphi\|_{H}^{2} .
\end{gathered}
$$

Hence, exploiting in the case $\delta>0$ the additional regularity assumption (3.22), we can use Gronwall's lemma in the above relation to deduce that $\left(\mathbf{u}_{1}, \varphi_{1}, \pi_{1}\right)$ coincides with $\left(\mathbf{u}_{2}, \varphi_{2}, \pi_{2}\right)$ over the whole of $(0, T)$, which actually concludes the proof of the theorem.

Remark 7. It is clear that, in the case when the initial data for $\left(\mathbf{u}_{1}, \varphi_{1}, \pi_{1}\right)$ and $\left(\mathbf{u}_{2}, \varphi_{2}, \pi_{2}\right)$ do not coincide with each other, then one can obtain from (4.96) a continuous dependence estimates in the norms specified in (3.14). Namely, one has

$$
\begin{aligned}
& \left\|\mathbf{u}_{1}(t)-\mathbf{u}_{2}(t)\right\|_{H_{\mathrm{div}}}^{2}+\left\|\varphi_{1}(t)-\varphi_{2}(t)\right\|_{V}^{2}+\left\|\pi_{1}(t)-\pi_{2}(t)\right\|_{H}^{2} \\
& \quad \leq C(T)\left(\left\|\mathbf{u}_{1}(0)-\mathbf{u}_{2}(0)\right\|_{H_{\mathrm{div}}}^{2}+\left\|\varphi_{1}(0)-\varphi_{2}(0)\right\|_{V}^{2}+\left\|\pi_{1}(0)-\pi_{2}(0)\right\|_{H}^{2}\right)
\end{aligned}
$$

for any $t \in[0, T]$, with the constant $C(T)$ on the right-hand side depending on the "strong" norms of the two solutions specified in (3.15)-(3.17) (and in (3.22) in the case $\delta>0$ ).

\section{Galerkin approximation}

In this section we present a possible construction of a sequence $\left(\mathbf{u}_{n}, \varphi_{n}, \pi_{n}\right)$ of approximate solutions by means of a Faedo-Galerkin scheme. Since the procedure is rather standard and follows an approach already used for similar models (see, e.g., [32, p. 284] or [13]), we will only give some highlights leaving the remaining details to the reader.

That said, we let $H_{\mathrm{div}, 0}$ and $V_{\mathrm{div}, 0}$ denote the subspaces, respectively, of $H_{\mathrm{div}}$ and of $V_{\mathrm{div}}$, consisting of the functions having zero spatial mean. Then we can define the Stokes operator as an unbounded linear operator on $H_{\text {div }, 0}$ by setting $A=-P \Delta, D(A)=H^{2}(\Omega) \cap V_{\text {div }, 0}$, where $P: L^{2}(\Omega) \rightarrow$ $H_{\text {div }}$ is the Leray projector 35 . Notice that

$$
(A \mathbf{u}, \mathbf{v})=((\mathbf{u}, \mathbf{v}))=(\nabla \mathbf{u}, \nabla \mathbf{v}) \quad \forall \mathbf{u} \in D(A), \quad \forall \mathbf{v} \in V_{\operatorname{div}, 0}
$$

Moreover, the operator $A$ is positive and self-adjoint on $H_{\text {div }, 0}$. Hence, we can take as a Galerkin base of $H_{\mathrm{div}, 0}$ the family $\left\{\mathbf{w}_{i}\right\}_{i \in \mathbb{N}}$ of the (linearly independent and properly normalized) eigenfunctions of $A$. Next, noting as $I$ the identity operator of $H$, we consider the unbounded linear operator $B$ of $H$ defined as $B:=I-\Delta$ with domain $D(B)=H^{2}(\Omega)$ (note that $\Omega$-periodicity is still implicitly assumed also at this level). Hence, $B$ is also positive and self-adjoint and we can take as a Galerkin base of $H$ the family $\left\{\tau_{i}\right\}_{i \in \mathbb{N}}$ of the (normalized and linearly independent) eigenfunctions of $B$. For any $m \in \mathbb{N}$, we can then define the $m$-dimensional subspaces $W_{m}:=\operatorname{span}\left\{\mathbf{w}_{1}, \ldots, \mathbf{w}_{m}\right\} \subset H_{\operatorname{div}, 0}$ and $T_{m}:=\operatorname{span}\left\{\tau_{1}, \ldots, \tau_{m}\right\} \subset H$. We also denote as $P_{m}: H_{\mathrm{div}, 0} \rightarrow W_{m}$ and $\Pi_{m}: H \rightarrow T_{m}$ the orthogonal projectors onto $W_{m}$ and $T_{m}$, respectively.

Finally, it is convenient to replace $f$ with a suitable regularization $f_{n}$ depending on a further approximation parameter $n$. More precisely, we assume that $f_{n} \in C^{1}(\mathbb{R})$ with

$$
\left|f_{n}(r)\right|+\left|f_{n}^{\prime}(r)\right| \leq C_{n} \quad \forall n \in \mathbb{N}, r \in \mathbb{R},
$$

with the constants $C_{n}>0$ of course going to infinity as $n \nearrow \infty$. An explicit expression of $f_{n}$ can be easily constructed by suitably truncating $f$ outside some bounded interval $I_{n}$ increasing with respect to $n$, for example $I_{n}=[-n, n]$. Then we obtain as a byproduct that $f_{n}$ converges to $f$ uniformly on compact sets of $\mathbb{R}$ as $n \nearrow \infty$.

With this machinery at hand, we can look for a Faedo-Galerkin solution of the form

$$
\mathbf{u}_{m}=\sum_{j=1}^{m} u_{j}^{m}(t) \mathbf{w}_{j}, \quad \varphi_{m}=\sum_{j=1}^{m} \varphi_{j}^{m}(t) \tau_{j}, \quad \pi_{m}=\sum_{j=1}^{m} \pi_{j}^{m}(t) \tau_{j},
$$


satisfying the following discretized system:

$$
\begin{aligned}
& \left(\partial_{t} \mathbf{u}_{m}, \mathbf{w}\right)+\left(\mathbf{u}_{m} \cdot \nabla \mathbf{u}_{m}, \mathbf{w}\right)+\left(A \mathbf{u}_{m}, \mathbf{w}\right)=\left(\nabla \varphi_{m} \otimes \nabla \varphi_{m}, \nabla \mathbf{w}\right), \quad \forall \mathbf{w} \in V_{m}, \\
& \pi_{m}=\partial_{t} \varphi_{m}+\Pi_{m}\left(\mathbf{u}_{m} \cdot \nabla \varphi_{m}\right)+\epsilon B \varphi_{m}+(\sigma-\epsilon) \varphi_{m}, \\
& \kappa \partial_{t} \pi_{m}+\pi_{m}+\delta \Pi_{m}\left(\mathbf{u}_{m} \cdot \nabla \pi_{m}\right)+B \varphi_{m}-\varphi_{m}+\Pi_{m}\left(f_{n}\left(\varphi_{m}\right)\right)=0,
\end{aligned}
$$

complemented with the initial conditions

$$
\left.\mathbf{u}_{m}\right|_{t=0}=P_{m} \mathbf{u}_{0},\left.\quad \varphi_{m}\right|_{t=0}=\Pi_{m} \varphi_{0},\left.\quad \pi_{m}\right|_{t=0}=\Pi_{m} \pi_{0} .
$$

Since all nonlinear terms in the above system have at least a locally Lipschitz dependence on their arguments, it turns out that existence of a local in time solution to (5.2)-(5.4) with the initial conditions (5.5) is a consequence of the classical Cauchy theorem for ODE's. It is also worth stressing that the above constructed approximate solution, despite being identified by the sole subscript $m$, depends in fact on both approximation parameters $n$ and $m$.

For any fixed $n \in \mathbb{N}$, we will now let $m$ tend to infinity so to obtain a solution $\left(\mathbf{u}_{n}, \varphi_{n}, \pi_{n}\right)$ depending only on $n$. To this aim, we just reproduce the a priori bounds obtained in the previous section working now on the Faedo-Galerkin scheme. Indeed, all the computations can be repeated just with some small technical difference mainly related to the presence of a regularized nonlinear function $f_{n}$ in place of the original $f$. The only point we would like to remark refers to the use of (5.3) to compute the product term now taking the form $\left(\Pi_{m}\left(f_{n}\left(\varphi_{m}\right)\right), \pi_{m}\right)$ (compare with (4.4)). Indeed, in the present setting this gives rise to the quantity $\left(\Pi_{m}\left(f_{n}\left(\varphi_{m}\right)\right), \Pi_{m}\left(\mathbf{u}_{m} \cdot \nabla \varphi_{m}\right)\right)$ which does not necessarily vanish because both factors are projected onto the finite dimensonal subspace $T_{m}$. On the other hand, we can easily see that

$$
\begin{aligned}
\left(\Pi_{m}\left(f_{n}\left(\varphi_{m}\right)\right), \Pi_{m}\left(\mathbf{u}_{m} \cdot \nabla \varphi_{m}\right)\right) & =\left(\Pi_{m}\left(f_{n}\left(\varphi_{m}\right)\right), \mathbf{u}_{m} \cdot \nabla \varphi_{m}\right) \\
& =\left(\Pi_{m}\left(f_{n}\left(\varphi_{m}\right)\right)-f_{n}\left(\varphi_{m}\right), \mathbf{u}_{m} \cdot \nabla \varphi_{m}\right) \\
& \leq c_{n}\left\|\mathbf{u}_{m}\right\|_{H_{\mathrm{div}}}\left\|\nabla \varphi_{m}\right\|_{H},
\end{aligned}
$$

where we also used incompressibility and condition (5.1). Note that the additional contribution on the right-hand side, at fixed $n \in \mathbb{N}$, can be controlled using Gronwall (giving rise to an additional quantity in the approximate energy inequality). On the other hand, as we take $m \nearrow \infty$, the projection operators disappear and we do no longer face any additional term when repeating the estimates on $\left(\mathbf{u}_{n}, \varphi_{n}, \pi_{n}\right)$.

It is also worth observing that, as we take $m \nearrow \infty$, we can rely on a set of a priori estimates that are uniform with respect to the time variable. Hence, as a consequence of standard extension arguments it will turn out that, despite Galerkin solutions $\left(\mathbf{u}_{m}, \varphi_{m}, \pi_{m}\right)$ might be defined only on small time intervals, the solutions $\left(\mathbf{u}_{n}, \varphi_{n}, \pi_{n}\right)$ obtained in the limit can be thought to be defined for every $t \in(0, \infty)$. As a consequence, we can take $\left(\mathbf{u}_{n}, \varphi_{n}, \pi_{n}\right)$ as a regularized solution to our system depending on the sole parameter $n$. Moreover, the a priori estimates obtained before are also uniform with respect to $n \in \mathbb{N}$. Hence, to let $n \nearrow \infty$, we can proceed as described in Subsection 4.3, with the sole differences related to the occurrence of the regularized nonlinearity $f_{n}$. Nevertheless, even here, the needed modifications are almost straightforward. Indeed, using the strong (hence a.e. pointwise) convergence of $\varphi_{n}$ (cf. (4.52)) and the fact that $f_{n} \rightarrow f$ uniformly on compact subsets of $\mathbb{R}$, it is easy to realize that, in place of (4.56), there holds

$$
f_{n}\left(\varphi_{n}\right) \rightarrow f(\varphi) \quad \text { weakly star in } L^{\infty}\left(0, T ; L^{\frac{p+2}{p+1}}(\Omega)\right) \text { and strongly in } L^{1}\left(0, T ; L^{1}(\Omega)\right) .
$$

Then, the rest of the argument works up to minor adaptations.

\section{Conclusive remarks}

In this part, we provide some additional considerations on the studied model and in particular we discuss some of the choices on coefficients and regularizing terms we operated for the purpose of obtaining a tractable mathematical problem. 


\subsection{The model without regularizing terms}

Our system (1.1)-(1.4) can be seen as a regularized version of a more basic model which can be written as follows:

$$
\begin{aligned}
& \mathbf{u}_{t}+\mathbf{u} \cdot \nabla \mathbf{u}+\nabla p-\Delta \mathbf{u}=-\operatorname{div}(\nabla \varphi \otimes \nabla \varphi) \\
& \operatorname{div} \mathbf{u}=0 \\
& \pi=\pi_{0}:=\varphi_{t}+\mathbf{u} \cdot \nabla \varphi \\
& \kappa\left(\pi_{t}+\mathbf{u} \cdot \nabla \pi\right)+\pi-\Delta \varphi+f(\varphi)=0
\end{aligned}
$$

The above system corresponds to (1.1)-(1.4) with the choices $\sigma=\epsilon=0$ and $\kappa=\delta$. It is worth noting that, neglecting the role of the velocity, i.e., taking $\mathbf{u}=0$, (6.3)-(6.4) reduce to the damped wave equation (or, equivalently, to the hyperbolic relaxation of the Allen-Cahn equation with "small" relaxation coefficient $\kappa$ ). When the contribution of $\mathbf{u}$ is included, proceeding as in Subsec. 4.1 one can easily obtain that (hypothetical) solutions to (6.1)-(6.4) satisfy the energy law

$$
\begin{gathered}
\frac{\mathrm{d}}{\mathrm{d} t} \mathcal{E}+\mathcal{D}_{0}=0, \quad \text { where } \\
\mathcal{E}=\frac{1}{2}\|\mathbf{u}\|_{H_{\mathrm{div}}}^{2}+\frac{\kappa}{2}\|\pi\|_{H}^{2}+\frac{1}{2}\|\nabla \varphi\|_{H}^{2}+\int_{\Omega} F(\varphi) \mathrm{d} x, \quad \mathcal{D}_{0}=\|\nabla \mathbf{u}\|_{H_{\mathrm{div}}}^{2}+\|\pi\|_{H}^{2} .
\end{gathered}
$$

Physically speaking the above relation states that the derivative of the energy $\mathcal{E}$ is given by minus the dissipation terms $\mathcal{D}_{0}$. Moreover, the four summands in the energy can be respectively interpreted as the kinetic-macroscopic, kinetic-microscopic, interface, and configuration energies. On the other hand, the two summands in $\mathcal{D}_{0}$ represent the dissipation due to viscosity effects in the flow and to the process of phase transition, respectively. Relation (6.5) represents the First Principle of Thermodynamics, stating that, in absence of external sources, a part of the energy $\mathcal{E}$ is gradually converted into heat. In particular, one obtains the finiteness of the dissipation integrals:

$$
\int_{0}^{+\infty} \mathcal{D}_{0}(t) \mathrm{d} t<+\infty .
$$

Remark 8 (nonisothermal case). If one wants to include temperature into the model, a nonisothermal version of (6.1)-(6.4) could be written, following the lines, e.g., of [16], in the form

$$
\begin{aligned}
& \mathbf{u}_{t}+\mathbf{u} \cdot \nabla \mathbf{u}+\nabla p-\Delta \mathbf{u}=-\operatorname{div}(\nabla \varphi \otimes \nabla \varphi), \\
& \operatorname{div} \mathbf{u}=0, \\
& \pi=\pi_{0}:=\varphi_{t}+\mathbf{u} \cdot \nabla \varphi, \\
& \kappa\left(\pi_{t}+\mathbf{u} \cdot \nabla \pi\right)+\pi-\Delta \varphi+f(\varphi)=\vartheta, \\
& \vartheta_{t}+\mathbf{u} \cdot \nabla \vartheta+\pi \vartheta-\operatorname{div}(a(\vartheta) \nabla \vartheta)=D_{0}:=|\nabla \mathbf{u}|^{2}+|\pi|^{2},
\end{aligned}
$$

where $\vartheta>0$ is the absolute temperature and $a(\vartheta)>0$ represents the (possibly constant) heat conductivity. Of course other nonisothermal effects may be also included in (6.8)-(6.12), like for instance a temperature dependent viscosity in (6.8). In particular the model written above is just the simplest one having some physical significance. In this setting, if we repeat the procedure leading to the energy inequality but add now also the integral of (6.12) over $\Omega$, noting that the integral of $D_{0}$ equals $\mathcal{D}_{0}$, we obtain (still for periodic boundary conditions) the modified energy balance

$$
\frac{\mathrm{d}}{\mathrm{d} t}\left(\mathcal{E}+\int_{\Omega} \vartheta \mathrm{d} x\right)=0
$$

where now the energy is conserved in view of the fact that also thermal energy is now considered. In addition to that, testing (6.12) by $-\vartheta^{-1}$ one obtains the entropy production inequality corresponding to the validity of the Second Principle of Thermodynamics.

It is finally worth observing that relation (6.5) (or its integral version (6.7)) prescribes that some amount of energy is converted into heat, but in itself it does not suffice to quantify the amount 
of energy that is released. In other words it does not imply the uniform dissipativity property leading to existence of an absorbing set. Actually, to achieve this, we need to mimick the procedure in the proof in Subsec. 4.2 exploiting the fact that we have taken here $\delta=\kappa$. Actually, testing (6.3) by $\kappa \pi$, (6.4) by $\varphi$, and using incompressibility, we deduce

$$
\kappa \frac{\mathrm{d}}{\mathrm{d} t} \int_{\Omega} \pi \varphi \mathrm{d} x+\int_{\Omega} \pi \varphi \mathrm{d} x+(-\Delta \varphi+f(\varphi), \varphi)=\kappa\|\varphi\|^{2}
$$

Then, repeating the procedure given in Subsec. 4.2, i.e., summing (a positive multiple of) (6.14) to (6.5), one obtains uniform dissipativity. This fact suggests that, from the energetic viewpoint, the case $\delta=\kappa$ is physically more sound. In other words, the correct relaxation of the Allen-Cahn equation with transport effect should be obtained by adding the second material derivative of $\varphi$, as done in (6.4) (and not, for instance, just its second partial derivative $\varphi_{t t}$ ). Of course, this choice has a mathematical drawback, namely, estimating the term $\mathbf{u} \cdot \nabla \pi$ may be complicated, especially in the setting of strong solutions. This is exactly the issue which led us to consider also, in our mathematical results, the case when $\kappa>0$ and $\delta=0$.

\subsection{Introduction of regularizing terms}

In the previous subsection we have seen that system (6.1)- 6.4 complies with the basic laws of Thermodynamics. Moreover, at least in absence of external source terms, solution trajectories satisfy a uniform dissipation principle. In this sense, the above model can be seen as a physically reasonable and very natural extension of the usual models for two-phase fluids (like those considered, e.g., in [1, 2, 9, 13]). On the other hand, as explained in the introduction, system (6.1)-(6.4) seems impervious to a rigorous mathematical analysis, even in two dimensions of space. In other words, if one wants to prove (at least) existence of weak solutions, some modification is mandatory. Indeed, the simplest and more natural strategy to obtain a mathematically tractable PDE system consists in the addition of regularizing terms, and this is what we did for the sake of proving our results. Of course, regularizing the model may affect the underlying physical aspects, and now we would like to discuss a bit this issue.

Since the main mathematical difficulties arise in connection with the regularity of $\varphi$, it appears to be natural to add regularizing terms either in (6.3) or in (6.4), and we actually made the first choice. Generally speaking, this corresponds to replacing (6.3) with

$$
\pi=\pi_{0}+\pi_{\text {reg }}, \quad \text { where, still, } \pi_{0}=\varphi_{t}+\mathbf{u} \cdot \nabla \varphi,
$$

and now $\pi_{\text {reg }}$ corresponds to the regularization term(s). The main reason for adding regularization terms in (6.3) (rather than, for instance, in (6.4) ) stands in the fact that this choice affects in a minimal way the other equations and, in particular, it does not generate (artificial) additional forces on the right-hand side of (6.1). Indeed, whatever is the choice of $\pi_{\text {reg, }}$, the strategy leading to the energy estimate (or, in other words, the variational structure of the system) remains essentially the same. Indeed, a simple check shows that (6.5) is now replaced by

$$
\frac{\mathrm{d}}{\mathrm{d} t} \mathcal{E}+\mathcal{D}_{0}+\mathcal{D}_{\text {reg }}=0, \quad \text { where } \mathcal{D}_{\text {reg }}=\left(\pi_{\text {reg }},-\Delta \varphi+f(\varphi)\right)
$$

and one needs to choose $\pi_{\text {reg }}$ in such a way that the additional dissipation $\mathcal{D}_{\text {reg }}$ has reasonable properties. In our setting, we actually took

$$
\pi_{\mathrm{reg}}=-\epsilon \Delta \varphi+\sigma \varphi
$$

in such a way that

$$
\mathcal{D}_{\text {reg }}=\epsilon\|\Delta \varphi\|^{2}+\int_{\Omega}\left(\sigma+\epsilon f^{\prime}(\varphi)\right)|\nabla \varphi|^{2} \mathrm{~d} x+\sigma \int_{\Omega} f(\varphi) \varphi \mathrm{d} x .
$$

Here the main issue stands in the nonconvexity of $F$ (or, equivalently, on the nonmonotonicity of $f$ ) implying that, particularly when $\sigma=0, \mathcal{D}_{\text {reg }}$ may be unbounded from below, (possibly) providing 
a growth, rather than a dissipation of energy in that case, i.e. a clearly nonphysical behavior (note, however, that in the case when $\kappa=\delta$, this issue may be somehow overcome as in Subsec. 4.2 or in (6.14)).

On the other hand, if $\epsilon$ and $\sigma$ are both strictly positive, even though the quantity $\mathcal{D}_{0}+\mathcal{D}_{\text {reg }}$ may be nonpositive (due to nonmonotonicity of $f$ ), it is however true that, up to a positive constant, $\mathcal{D}_{0}+\mathcal{D}_{\text {reg }}$ essentially controls the energy from above (namely, the above argument implies the inequality (4.17)). Hence, (6.18) in itself provides a uniform dissipativity principle, with no need of adding (6.14). For this reason, such a choice works also in the case when $\kappa \neq \delta$, i.e., (6.4) is replaced by

$$
\kappa \pi_{t}+\delta \mathbf{u} \cdot \nabla \pi+\pi-\Delta \varphi+f(\varphi)=0 .
$$

It is nevertheless true that, with the position (6.17), one loses the finiteness of the dissipation integrals (6.7) and, for this reason, in the case (6.17) the compatibility of the model with the Energy conservation principle cannot be considered to be complete. It is worth noting that, taking instead

$$
\pi_{\mathrm{reg}}=\epsilon(-\Delta \varphi+f(\varphi)),
$$

we would have

$$
\mathcal{D}_{\text {reg }}=\epsilon\|-\Delta \varphi+f(\varphi)\|^{2}
$$

whence the occurrence of global dissipation integrals would be kept in this case:

$$
\int_{0}^{+\infty}\left(\mathcal{D}_{0}(t)+\mathcal{D}_{\operatorname{reg}(t)}\right) \mathrm{d} t<+\infty
$$

As noted in the introduction, the model with the choice (6.20) may be studied in a forthcoming work.

\subsection{Neglecting the second material derivative}

A second simplification we operated in order to prove our mathematical results (particularly those devoted to strong solutions) is given by the choice $\delta=0$, that corresponds to replacing (6.4) with

$$
\kappa \pi_{t}+\pi-\Delta \varphi+f(\varphi)=0
$$

As noted above, on the one hand this choice has a poor motivation from the physical point of view (we saw that relaxation should be provided through the material derivative of $\pi$, i.e., assuming $\kappa=\delta$ ). On the other hand, it can be justified, at least partially, as a linearization argument which leads to neglecting some terms that depend at least quadratically on some "small" quantities. Indeed, if expanded, the (neglected) second order transport term can be written as

$$
\delta \mathbf{u} \cdot \nabla \pi=\delta \mathbf{u} \cdot \nabla\left(\varphi_{t}+\mathbf{u} \cdot \nabla \varphi\right)
$$

and, at least for small flow velocity $\mathbf{u}$, the above quantity can be though to be negligible compared to other terms. This is of course an "ad-hoc" simplification, but it is a procedure commonly performed in the mathematical literature on phase transition models, the most striking example being probably given by non-isothermal phase-field model. In that framework most of the mathematical works are devoted to models that are linearized around the critical temperature losing in that case the validity of the Second Principle of Thermodynamics. This is the case, for instance, of the so-called "Caginalp phase-field model" [10 which is studied, along with its many variants, in a huge number of mathematical papers. This model can be obtained from other types of (thermodynamically consistent, but mathematically more difficult) models exactly by neglecting some quadratic terms depending on "small" quantities, see for instance [19] and the references therein.

Acknowledgments. The present paper benefits from the support of the Italian MIUR-PRIN Grant 2015PA5MP7 "Calculus of Variations" and of the GNAMPA (Gruppo Nazionale per l'Analisi Matematica, la Probabilità e le loro Applicazioni) of INdAM (Istituto Nazionale di Alta Matematica) for GS. GF has been supported by the Austrian Science Fund (FWF) grant W1245 and by Vienna Doctoral School of Mathematics. 


\section{References}

[1] H. Abels, On a diffuse interface model for two-phase flows of viscous, incompressible fluids with matched densities, Arch. Ration. Mech. Anal., 194 (2009), 463-506.

[2] H. Abels and M. Röger, Existence of weak solutions for a non-classical sharp interface model for a two-phase flow of viscous, incompressible fluids, Ann. Inst. H. Poincaré Anal. Non Linéaire, 26 (2009), 2403-2424.

[3] S.M. Allen and J.W. Cahn, A microscopic theory for antiphase boundary motion and its application to antiphase domain coarsening, Acta Metallurgica, 27 (1979), 1085-1095.

[4] D.M. Anderson, G.B. McFadden and A.A. Wheeler, Diffuse-interface methods in fluid mechanics, Annu. Rev. Fluid Mech., Palo Alto, CA, Vol. 30, 1998, 139-165.

[5] V. Barbu, I. Lasiecka and M.A. Rammaha, On nonlinear wave equations with degenerate damping and source terms, Trans. Amer. Math. Soc., 357 (2005), 2571-2611.

[6] T. Blesgen, A generalization of the Navier-Stokes equation to two-phase flows, J. Physics D (Applied Physics), 32 (1999), 1119-1123.

[7] L. Bociu and I. Lasiecka, Uniqueness of weak solutions for the semilinear wave equations with supercritical boundary/interior sources and damping, Discrete Contin. Dyn. Syst., 22 (2008), $835-860$.

[8] G. Bonfanti, M. Frémond and F. Luterotti, Existence and uniqueness results to a phase transition model based on microscopic accelerations and movements, Nonlinear Anal. Real World Appl., 5 (2004), 123-140.

[9] F. Boyer, Nonhomogeneous Cahn-Hilliard fluids, Ann. Inst. H. Poincaré Anal. Non Linéaire, 18 (2001), 225-259.

[10] G. Caginalp, An analysis of a phase field model of a free boundary, Arch. Rational Mech. Anal., 92 (1986), 205-245.

[11] C. Cao and C.G. Gal, Global solutions for the 2D NSCH model for a two-phase flow of viscous, incompressible fluids with mixed partial viscosity and mobility, Nonlinearity, 25 (2012), 32113234 .

[12] I. Chueshov and I. Lasiecka, Attractors for second-order evolution equations with a nonlinear damping, J. Dynam. Differential Equations, 16 (2004), 469-512.

[13] P. Colli, S. Frigeri and M. Grasselli, Global existence of weak solutions to a nonlocal CahnHilliard-Navier-Stokes system, J. Math. Anal. Appl., 386 (2012), 428-444.

[14] F. Dell'Oro and V. Pata, Long-term analysis of strongly damped nonlinear wave equations, Nonlinearity, 24 (2011), 3413-3435.

[15] F. Dell'Oro and V. Pata, Strongly damped wave equations with critical nonlinearities, Nonlinear Anal., 75 (2012), 5723-5735.

[16] M. Eleuteri, E. Rocca and G. Schimperna, On a non-isothermal diffuse interface model for twophase flows of incompressible fluids, Discrete Contin. Dyn. Syst., 35 (2015), 119-138.

[17] M. Eleuteri, E. Rocca and G. Schimperna, Existence of solutions to a two-dimensional model for nonisothermal two-phase flows of incompressible fluids, Ann. Inst. H. Poincaré Anal. Non Linéaire, 33 (2016), 1431-1454.

[18] E. Feireisl, E. Rocca, G. Schimperna and A. Zarnescu, On a hyperbolic system arising in liquid crystals modeling, J. Hyperbolic Differ. Equ., 15 (2018), 15-35. 
[19] E. Feireisl, H. Petzeltová, and E. Rocca, Existence of solutions to a phase transition model with microscopic movements, Math. Methods Appl. Sci., 32 (2009), 1345-1369.

[20] J. Feng, C. Liu, J. Shen and P. Yue, A diffuse-interface method for simulating two-phase flows of complex fluids, J. Fluid Mech., 515 (2004), 293-317.

[21] M. Frémond, Non-smooth Thermomechanics, Springer-Verlag, Berlin, 2002.

[22] F. Gay-Balmaz and C. Tronci, The helicity and vorticity of liquid-crystal flows, Proc. R. Soc. Lond. Ser. A Math. Phys. Eng. Sci., 467 (2011), 1197-1213.

[23] C.G. Gal and M. Grasselli, Asymptotic behavior of a Cahn-Hilliard-Navier-Stokes system in 2D, Ann. Inst. H. Poincaré Anal. Non Linéaire, 27 (2010), 401-436.

[24] M. Grasselli and V. Pata, Existence of a universal attractor for a parabolic-hyperbolic phase-field system, Adv. Math. Sci. Appl., 13 (2003), 443-459.

[25] M. Grasselli, A. Miranville, V. Pata and S. Zelik, Well-posedness and long time behavior of a parabolic-hyperbolic phase-field system with singular potentials, Math. Nachr., 280 (2007), 1475-1509.

[26] M. Grasselli, G. Schimperna and S. Zelik, On the 2D Cahn-Hilliard equation with inertial term, Comm. Partial Differential Equations, 34 (2009), 137-170.

[27] M.E. Gurtin, D. Polignone and J. Viñals, Two-phase binary fluids and immiscible fluids described by an order parameter, Math. Models Methods Appl. Sci., 6 (1996), 815-831.

[28] P.C. Hohenberg and B.I. Halperin, Theory of dynamic critical phenomena, Rev. Mod. Phys., 49 (1977), 435-479.

[29] A. Kh. Khanmamedov, Global attractors for strongly damped wave equations with displacement dependent damping and nonlinear source term of critical exponent, Discrete Contin. Dyn. Syst., 31 (2011), 2497-2522.

[30] I. Lasiecka and D. Toundykov, Energy decay rates for the semilinear wave equation with nonlinear localized damping and source terms, Nonlinear Anal., 64 (2006), 1757-1797.

[31] C. Liu and J. Shen, A phase field model for the mixture of two incompressible fluids and its approximation by a Fourier-spectral method, Phys. D, 179 (2003), 211-228.

[32] A. Miranville, Some mathematical models in phase transition, Discrete Contin. Dyn. Syst. Ser. S, 7 (2014), 271-306.

[33] J.C. Robinson, Infinite-Dimensional Dynamical Systems: An Introduction to Dissipative Parabolic PDEs and the Theory of Global Attractors. Cambridge University Press, 2001.

[34] L. Roder and T. Tébou, Stabilization of the wave equation with localized nonlinear damping, J. Differential Equations, 145 (1998), 502-524.

[35] R. Temam, Navier-Stokes Equations, North-Holland, Amsterdam, 1984.

[36] L. Zhao, H. Wu and H. Huang, Convergence to equilibrium for a phase-field model for the mixture of two viscous incompressible fluids, Commun. Math. Sci., 7 (2009), 939-962. 